\title{
Carbon solution and partitioning between metallic and silicate melts in a shallow magma ocean: Implications for the origin and distribution of terrestrial carbon
}

\author{
Rajdeep Dasgupta ${ }^{\mathrm{a}, *}$, Han Chi ${ }^{\mathrm{a}}$, Nobumichi Shimizu ${ }^{\mathrm{c}}$, Antonio S. Buono ${ }^{\mathrm{b}}$, \\ David Walker ${ }^{\text {b }}$ \\ ${ }^{a}$ Department of Earth Science, Rice University, MS 126, 6100 Main Street, Houston, TX 77005, United States \\ ${ }^{\mathrm{b}}$ Lamont-Doherty Earth Observatory, Columbia University, 61 Route $9 W$, Palisades, NY 10964, United States \\ ${ }^{\mathrm{c}}$ Department of Geology and Geophysics, Woods Hole Oceanographic Institution, Woods Hole, MA 02543, United States
}

Received 6 June 2012; accepted in revised form 6 October 2012; available online 22 October 2012

\begin{abstract}
The origin of bulk silicate Earth carbon inventory is unknown and the fate of carbon during the early Earth differentiation and core formation is a missing link in the evolution of the terrestrial carbon cycle. Here we present high pressure $(P)-$ temperature $(T)$ experiments that offer new constraints upon the partitioning of carbon between metallic and silicate melt in a shallow magma ocean. Experiments were performed at $1-5 \mathrm{GPa}, 1600-2100{ }^{\circ} \mathrm{C}$ on mixtures of synthetic or natural silicates (tholeiitic basalt/alkali basalt/komatiite/fertile peridotite) and $\mathrm{Fe}-\mathrm{Ni}-\mathrm{C} \pm \mathrm{Co} \pm \mathrm{S}$ contained in graphite or $\mathrm{MgO}$ capsules. All the experiments produced immiscible Fe-rich metallic and silicate melts at oxygen fugacity $\left(f \mathrm{O}_{2}\right)$ between $\sim \mathrm{IW}-1.5$ and IW1.9. Carbon and hydrogen concentrations of basaltic glasses and non-glassy quenched silicate melts were determined using secondary ionization mass spectrometry (SIMS) and speciation of dissolved C-O-H volatiles in silicate glasses was studied using Raman spectroscopy. Carbon contents of metallic melts were determined using both electron microprobe and SIMS. Our experiments indicate that at core-forming, reduced conditions, carbon in deep mafic-ultramafic magmas may dissolve primarily as various hydrogenated species but the total carbon storage capacity, although is significantly higher than solubility of $\mathrm{CO}_{2}$ under similar conditions, remains low $(<500 \mathrm{ppm})$. The total carbon content in our reduced melts at graphite saturation increases with increasing melt depolymerization $(\mathrm{NBO} / \mathrm{T})$, consistent with recent spectroscopic studies, and modestly with increasing hydration. Carbon behaves as a metal-loving element during core-mantle separation and our experimental $D_{\mathrm{C}}^{\mathrm{metal} / \text { silicate }}$ varies between $\sim 4750$ and $\geqslant 150$ and increases with increasing pressure and decreases with increasing temperature and melt NBO/T.

Our data suggest that if only a trace amount of carbon $(\sim 730 \mathrm{ppm} C)$ was available during early Earth differentiation, most of it was partitioned to the core (with $0.20-0.25 \mathrm{wt} . \% \mathrm{C}$ ) and no more than $\sim 10-30 \%$ of the present-day mantle carbon budget (50-200 ppm $\mathrm{CO}_{2}$ ) could be derived from a magma ocean residual to core formation. With equilibrium core formation removing most of the carbon initially retained in the terrestrial magma ocean, explanation of the modern bulk silicate Earth carbon inventory requires a later replenishment mechanism. Partial entrapment of metal melt in solid silicate matrix, carbon ingassing by magma ocean-atmosphere interaction, and carbon outgassing from the core aided by reaction of core metal and deeply subducted water are some of the viable mechanisms.
\end{abstract}

(C) 2012 Elsevier Ltd. All rights reserved.

\footnotetext{
* Corresponding author. Tel.: +1 7133482664 .

E-mail address: Rajdeep.Dasgupta@rice.edu (R. Dasgupta).
} 


\section{INTRODUCTION}

The cycle of carbon through Earth's deep interior is important for chemical differentiation, internal dynamics, interior-exosphere exchange of trace gases, and long-term climate. Although a number of studies have considered the deep carbon cycle in the plate-tectonic framework of the modern Earth, little is known about the origin of deep Earth carbon. Since how long has the Earth's mantle possessed the carbon budget that we observe today? Is the present-day inventory of mantle carbon set by the balance of the modern Earth processes such as recycling of crustal materials and volcanic degassing, or was the budget set early in the Earth's history, even before the initiation of plate tectonics? If the latter, does the terrestrial carbon to some extent date back to the Hadean eon and was it affected by the early Earth differentiation and core formation? The relative contribution of modern versus ancient processes is key in unraveling the origin of terrestrial carbon, i.e., whether the mantle budget is dominantly primordial or recycled.

In order to constrain the origin of deep Earth carbon, the element's behavior, abundance, and distribution during planetary accretion and early planetary differentiation needs to be known. In particular, the magma ocean's storage capacity and carbon fractionation between metal and silicate during core formation, is largely unconstrained and has been a subject of interest in a number of recent studies (Kuramoto and Matsui, 1996; Kuramoto, 1997; Dasgupta and Walker, 2008; Hirschmann and Dasgupta, 2009; Dasgupta and Hirschmann, 2010). If carbon was available and took part in core-forming processes then its solution and partitioning behavior between metallic and silicate melt likely had a tremendous influence on the inventory and origin of deep Earth carbon. For example, if carbon partitioned strongly into the segregating core liquid, then it could be a dominant and important light element in the core. On the other hand, if silicate magma retained sufficient carbon in solution even in the strongly reduced condition set by the presence of a Fe-rich metallic melt (e.g., Walter and Tronnes, 2004; Wood et al., 2006) then such carbon content may largely set the mantle budget of carbon. It is therefore important to quantify the partitioning behavior of carbon between coexisting metallic and silicate melts at the physical and chemical conditions of their separation, i.e., in a magma ocean.

The separation of metal and silicate, to form a metallic core and a silicate mantle, in the early Earth is thought to have largely taken place in magma oceans (e.g., Stevenson, 1981; Walter and Tronnes, 2004; Wood et al., 2006). Extensive melting of the Earth and the formation of a deep magma ocean as a consequence of more than one giant impact, and perhaps numerous smaller impacts in between, facilitated metal-silicate equilibration and separation. Fractionation of elements (e.g., siderophile elements) between metallic core and silicate mantle during core formation is largely responsible for setting the elemental distribution in the terrestrial reservoirs (e.g., Li and Agee, 1996; Jana and Walker, 1997; Chabot et al., 2005; Wood et al., 2006; Righter, 2011; Rubie et al., 2011). The same fractionation process likely also has influenced the bulk distribution of the terrestrial volatiles in general and carbon in particular (Kuramoto and Matsui, 1996; Kuramoto, 1997; Dasgupta and Walker, 2008). If the entire budget of the present-day bulk Earth carbon participated in the metal-silicate fractionation and if $D_{\mathrm{C}}^{\mathrm{metal} / \text { silicate }}$ (partition coefficient of carbon between metal and silicate $=$ concentration of carbon in metal melt/concentration of carbon in silicate melt) is $<1$ then we expect the carbon to be concentrated mostly in the outer silicate layer, on the other hand, if $D_{\mathrm{C}}^{\mathrm{metal} / \text { silicate }} \gg 1$ then bulk carbon of the planet might be mostly sequestered into the core and hence may have much less impact in the long-term carbon cycle - assuming a limited return of carbon from the core to the mantle since then. Moreover, even if carbon preferentially partitioned into the core-forming liquid, did the Earth's molten silicate manage to retain enough carbon in dissolved form to explain the present-day carbon budget of the Earth's mantle or was a late volatile-rich veneer or other post-core segregation processes, such as early planetary atmosphere-magma ocean interaction, responsible for bringing carbon to the planet?

In order to constrain the fate of carbon during coremantle equilibration in a magma ocean, we performed high $P-T$ experiments that simulate $\mathrm{Fe}-\mathrm{Ni}$ metallic melt and mafic-ultramafic silicate melt equilibration. Our experiments were designed to explore the effect of silicate melt composition and pressure on $D_{\mathrm{C}}^{\mathrm{metal} / \text { silicate }}$, at two distinct temperatures and a narrow range of oxygen fugacity. Our measured partition coefficients for carbon suggest that the current estimate of mantle carbon is too high to be matched by carbon that is left behind in the residual silicate melt after core formation. Consideration of post-core formation processes to replenish the mantle carbon is necessary.

\section{METHODS}

\subsection{Experimental techniques}

\subsubsection{Starting mixes and capsule materials}

All the experiments were performed with homogeneous mixtures of silicates and 30-35 wt.\% Fe-rich metal mixes in graphite or crushable $\mathrm{MgO}$ capsules. Five different silicate mixes were used - a synthetic tholeiite (ThB), a natural mid-Atlantic ridge basalt (MAR), a natural alkalic basalt (Knippa), a natural komatiite (MT-7C), and a natural spinel peridotite xenolith (KLB-1) (Table 1). The range of silicate melt compositions was chosen to explore the possible role of magma ocean composition on the dissolution and metal-silicate partitioning of carbon. The range of basalt compositions also allowed us to constrain the possible role of melt compositions on carbon content at graphite saturation [CCGS] only from glassy samples and thus to evaluate whether the measured carbon content for non-glassy quenched matte (from peridotite, komatiite, and from experiments conducted in $\mathrm{MgO}$ capsules) can reliably be used (see more details in the Section 2.2). The compositions, in terms of $\mathrm{NBO} / \mathrm{T}$ (molar ratio of non-bridging oxygens per tetrahedrally coordinated cations; Mysen, 1988), were 0.74 for ThB, 0.80 for MAR, 1.40 for Knippa, 1.85 for MT-7C, and 2.74 for KLB-1. All the silicate compositions 
Table 1

Compositions of silicate and metal fractions in the experimental starting mixes.

\begin{tabular}{|c|c|c|c|c|c|c|c|c|c|c|c|c|c|c|}
\hline Silicates & $\mathrm{SiO}_{2}$ & $\mathrm{TiO}_{2}$ & $\mathrm{Al}_{2} \mathrm{O}_{3}$ & $\mathrm{Cr}_{2} \mathrm{O}_{3}$ & $\mathrm{FeO}^{*}$ & $\mathrm{MnO}$ & $\mathrm{MgO}$ & $\mathrm{NiO}$ & $\mathrm{CoO}$ & $\mathrm{CaO}$ & $\mathrm{Na}_{2} \mathrm{O}$ & $\mathrm{K}_{2} \mathrm{O}$ & $\mathrm{P}_{2} \mathrm{O}_{5}$ & $\begin{array}{l}\mathrm{NBO} / \\
\mathrm{T}\end{array}$ \\
\hline ThB & 50.5 & $\begin{array}{l}0.93 \\
(3)\end{array}$ & $\begin{array}{l}15.9 \\
(3)\end{array}$ & $\begin{array}{l}0.03 \\
(2)\end{array}$ & $\begin{array}{l}9.06 \\
(17)\end{array}$ & $\begin{array}{l}0.23 \\
(3)\end{array}$ & $\begin{array}{l}8.63 \\
(13)\end{array}$ & - & - & $\begin{array}{l}11.2 \\
(1)\end{array}$ & $\begin{array}{l}3.01 \\
(3)\end{array}$ & $\begin{array}{l}0.10 \\
(3)\end{array}$ & - & 0.75 \\
\hline MAR & $\begin{array}{l}49.36 \\
(9)\end{array}$ & $\begin{array}{l}1.27 \\
(5)\end{array}$ & $\begin{array}{l}14.90 \\
(4)\end{array}$ & & $\begin{array}{l}10.55 \\
(23)\end{array}$ & $\begin{array}{l}0.30 \\
(1)\end{array}$ & $\begin{array}{l}9.14 \\
(01)\end{array}$ & $\begin{array}{l}0.06 \\
(1)\end{array}$ & $\begin{array}{l}0.14 \\
(0)\end{array}$ & $\begin{array}{l}10.80 \\
(4)\end{array}$ & $\begin{array}{l}2.43 \\
(7)\end{array}$ & $\begin{array}{l}0.09 \\
(1)\end{array}$ & - & 0.80 \\
\hline Knippa & $40.3(1)$ & $\begin{array}{l}3.34 \\
(1)\end{array}$ & $\begin{array}{l}11.0 \\
(2)\end{array}$ & $\begin{array}{l}0.07 \\
(0)\end{array}$ & $\begin{array}{l}11.55 \\
(9)\end{array}$ & $\begin{array}{l}0.19 \\
(1)\end{array}$ & $\begin{array}{l}13.75 \\
(9)\end{array}$ & - & - & $\begin{array}{l}11.82 \\
(6)\end{array}$ & $\begin{array}{l}3.17 \\
(5)\end{array}$ & $\begin{array}{l}1.79 \\
(3)\end{array}$ & $\begin{array}{l}0.81 \\
(1)\end{array}$ & 1.40 \\
\hline $\begin{array}{l}\text { MT-7C } \\
\text { KLB-1 }\end{array}$ & $\begin{array}{l}46.00 \\
44.84 \\
(23)\end{array}$ & $\begin{array}{l}0.35 \\
0.11 \\
(2)\end{array}$ & $\begin{array}{l}7.61 \\
3.51 \\
(5)\end{array}$ & $\begin{array}{l}0.34 \\
0.32 \\
(4)\end{array}$ & $\begin{array}{l}10.40 \\
8.20 \\
(12)\end{array}$ & $\begin{array}{l}0.17 \\
0.12 \\
(3)\end{array}$ & $\begin{array}{l}25.80 \\
39.52 \\
(36)\end{array}$ & - & - & $\begin{array}{l}7.45 \\
3.07 \\
(3)\end{array}$ & $\begin{array}{l}0.51 \\
0.30 \\
(3)\end{array}$ & $\begin{array}{l}0.11 \\
0.02 \\
(1)\end{array}$ & $\begin{array}{l}- \\
-\end{array}$ & $\begin{array}{l}1.85 \\
2.74\end{array}$ \\
\hline Metals & $\mathrm{Fe}$ & $\mathrm{Ni}$ & $\mathrm{Co}$ & $\mathrm{C}$ & $\mathrm{S}$ & & & & & & & & & \\
\hline $\begin{array}{l}\text { Core } 1 \\
\text { Core } 2 \\
\text { Core } 3\end{array}$ & $\begin{array}{l}88.49 \\
89.63 \\
94.40\end{array}$ & $\begin{array}{l}5.38 \\
7.02 \\
5.60\end{array}$ & $\begin{array}{l}3.08 \\
0.20\end{array}$ & $\begin{array}{l}1.03 \\
1.09\end{array}$ & $\begin{array}{l}2.01 \\
2.06\end{array}$ & & & & & & & & & \\
\hline
\end{tabular}

'ThB' - a tholeiitic basalt synthesized using reagent grade oxides and carbonates and decarbonated and dried at $f \mathrm{O}_{2}$ of $\sim \mathrm{FMQ}-2$ at 1 atmosphere; the reported major element composition is based on electron probe analysis of a glass made using a piston cylinder device. 'MAR' - a natural Mid-Atlantic Ridge basalt; the composition is reported based on electron probe analysis of an experimentally synthesized glass using piston cylinder device. 'Knippa' - a natural alkalic basalt from the study of Lewis et al. (1993); the composition reported here is based on electron microprobe analysis of an experimentally synthesized glass using piston cylinder device. 'MT-7C' - a natural komatiite sample from Munro Township in the Abitibi belt of Canada; the composition is reported from Cottrell and Walker (2006). 'KLB-1' - a natural fertile, spinel peridotite xenolith from Kilbourne Hole, New Mexico, USA; the composition is reported from Davis et al. (2009). The numbers in parentheses, where present, indicate $1 \sigma$ standard deviation based on replicate electron microprobe analyses and are reported as last digit cited; 50.5(3) should be read as $50.5 \pm 0.3 \mathrm{wt} . \%$.

were finely powdered and reduced and dehydrated in a oneatmosphere gas mixing furnace between FMQ and IW buffer. Two experiments with Knippa alkalic basalt were conducted with unfired natural composition to constrain the effect of possibly higher dissolved water in the silicate on carbon solution and CCGS. This last composition will hereafter be referred to as Knippa-H.

Three different metal mixes - 'core 1', 'core 2', and 'core 3 ' - in the system $\mathrm{Fe}-\mathrm{Ni}-\mathrm{C} \pm \mathrm{Co} \pm \mathrm{S}$ were used with the first comprising wt.\% level, the second minor Co content, and the third no Co (Table 1). The composition 'core 1' and 'core 2' also contained $\sim 2$ wt.\% S, concentration small enough to not cause immiscibility in the $\mathrm{Fe}-\mathrm{Ni}-\mathrm{C}-\mathrm{S}$ metallic melt over the pressure range investigated (Dasgupta et al., 2009). Metal compositions 'core 1' and 'core 2' were prepared by mixing reagent grade metals, diamond, and laboratory synthesized FeS. FeS synthesis procedure is reported in Dasgupta et al. (2009). Metal composition 'core 3 ' run in graphite capsules was prepared by mixing reagent grade $\mathrm{Fe}$ and Ni powders. Homogeneous mixtures of metallic and silicate mixes were finally prepared using an agate mortar and pestle, under acetone. The dried metal-silicate mixtures were stored in a desiccator.

Most of the experiments reported here were performed in graphite capsules with a limited number performed in crushable, polycrystalline $\mathrm{MgO}$ capsules (Table 2). The choice of graphite as a capsule material allowed us to constrain bulk carbon solubility at graphite saturation in silicate melt at our experimental conditions. It also allowed us to add to the growing measurements of carbon solubility in core-forming alloy melt (Dasgupta and Walker, 2008;
Nakajima et al., 2009) at magma ocean conditions. However, the use of graphite as sample containers also forced saturation of carbon in the two melts, which likely is not realistic from the standpoint of a magma ocean carbon cycle. We note, however, that although the absolute concentrations of carbon in the two coexisting melts provide maxima, the partitioning between metallic and silicate melts should be independent of the $\mathrm{C}$ content of the bulk system. The rationale of using $\mathrm{MgO}$ capsules was two fold - (a) to try to constrain the partitioning behavior of carbon in graphite-undersaturated conditions and (b) to test whether reacted silicate melt compositions that likely yield heterogeneous aggregate of quench crystals can be analyzed reliably for carbon concentrations using ion microprobe technique.

The coexistence of Fe-rich metallic melt and maficultramafic silicate melt constrained the experimental $f \mathrm{O}_{2}$ via the equilibrium:

$$
\underset{\text { (silicate melt) }}{\mathrm{FeO}}=\underset{\text { (metal alloy melt) }}{\mathrm{Fe}}+0.5 \mathrm{O}_{2}
$$

where the $f \mathrm{O}_{2}$ of the experiment can be expressed relative to the $f \mathrm{O}_{2}$ of the iron-wüstite buffer, as:

$2 \log \left(a_{\mathrm{FeO}} / a_{\mathrm{Fe}}\right)=2 \log \left(X_{\mathrm{FeO}} \cdot \gamma_{\mathrm{FeO}} / X_{\mathrm{Fe}} \cdot \gamma_{\mathrm{Fe}}\right)=\Delta \mathrm{IW}$

Experimental $f \mathrm{O}_{2} \mathrm{~S}$ relative to the iron-wüstite buffer were calculated assuming a non-ideal solution model for both metallic and silicate liquid (Table 2). A fixed $\gamma_{\mathrm{FeO}}$ value of 1.5 , the average from the two studies that determined $\gamma_{\mathrm{FeO}}$ for a wide range of melt compositions were used (Holzheid et al., 1997; O'Neill and Eggins, 2002), assuming no significant pressure effect on $\gamma_{\mathrm{FeO}}$ (Toplis, 2005). Activity 
Table 2

Summary of experimental run conditions.

\begin{tabular}{|c|c|c|c|c|c|c|c|c|c|}
\hline Run No. & Silicate/metal composition & $P(\mathrm{GPa})$ & $T\left({ }^{\circ} \mathrm{C}\right)$ & Capsule material & $f \mathrm{O}_{2}(\Delta \mathrm{IW})^{\mathrm{a}}$ & $f \mathrm{O}_{2}(\Delta \mathrm{IW})^{\mathrm{b}}$ & $f \mathrm{O}_{2}(\Delta \mathrm{IW})^{\mathrm{c}}$ & $\mathrm{NBO} / \mathrm{T}$ & Silicate melt texture \\
\hline B109 & $\mathrm{ThB} / 30 \%$ core 3 & 1 & 1600 & Graphite & -1.49 & -1.47 & -1.56 & 0.85 & Glass \\
\hline $\mathrm{G} 212$ & $\mathrm{ThB} / 30 \%$ core 3 & 3 & 1600 & Graphite & -1.50 & -1.53 & -1.54 & 0.84 & Glass \\
\hline B172 & Knippa $30 \%$ core 3 & 1 & 1600 & Graphite & -1.49 & -1.48 & -1.56 & 1.51 & Glass \\
\hline B170 & Knippa $/ 30 \%$ core 3 & 3 & 1600 & Graphite & -1.46 & -1.50 & -1.50 & 1.44 & Glass \\
\hline B149 & Knippa-H/30\% core 3 & 1 & 1600 & Graphite & -1.45 & -1.51 & -1.48 & 1.58 & Glass \\
\hline B148 & Knippa-H/30\% core 3 & 3 & 1600 & Graphite & -1.57 & -1.68 & -1.57 & 1.46 & Glass \\
\hline BB-932 & MAR $/ 35 \%$ core 2 & 2 & 2000 & Graphite & -1.66 & -1.64 & -1.67 & 0.84 & Glass \\
\hline BB-929 & MAR $/ 35 \%$ core 2 & 3 & 2000 & Graphite & -1.66 & -1.64 & -1.67 & 0.86 & Glass \\
\hline BB-930 & $\mathrm{MAR} / 35 \%$ core 2 & 4 & 2000 & Graphite & -1.67 & -1.70 & -1.64 & 0.85 & Glass \\
\hline BB-914 & MT-7C/30\% core 1 & 2 & 2000 & Graphite & -1.73 & -1.77 & -1.69 & 1.92 & Glass \\
\hline BB-905 & MT-7C/30\% core 1 & 3 & 2000 & Graphite & -1.82 & -1.87 & -1.77 & 2.65 & Dendritic \\
\hline BB-904 & MT-7C/30\% core 1 & 4 & 2000 & Graphite & -1.72 & -1.81 & -1.66 & 2.65 & Dendritic \\
\hline BB-901 & MT-7C/30\% core 1 & 5 & 2000 & Graphite & -1.73 & -1.85 & -1.66 & 1.98 & Dendritic \\
\hline BB-919 & KLB- $1 / 35 \%$ core 1 & 3 & 2000 & Graphite & -1.68 & -1.80 & -1.61 & 2.83 & Dendritic \\
\hline BB-920 & KLB- $1 / 35 \%$ core 1 & 4 & 2000 & Graphite & -1.84 & -1.93 & -1.78 & 2.59 & Dendritic \\
\hline BB-916 & KLB- $1 / 35 \%$ core 1 & 5 & 2000 & Graphite & -1.75 & -1.92 & -1.66 & 2.13 & Dendritic \\
\hline BB-918 & MT $-7 \mathrm{C} / 30 \%$ core 1 & 3 & 2000 & $\mathrm{MgO}$ & & -1.78 & -1.93 & 3.33 & Dendritic \\
\hline BB-921 & MT- $7 \mathrm{C} / 30 \%$ core 1 & 3 & 2100 & $\mathrm{MgO}$ & & -1.75 & -1.99 & 3.58 & Dendritic \\
\hline BB-940 & MAR $/ 35 \%$ core 2 & 4 & 2000 & $\mathrm{MgO}$ & & -1.87 & -2.17 & 2.64 & Dendritic \\
\hline
\end{tabular}

" Experiments performed using piston cylinder (PC) device are prefixed with 'B' or ' $\mathrm{G}$ ' and those using a multi anvil (MA) device with 'BB'. PC experiments were run for $2 \mathrm{~h}$ and $\mathrm{MA}$ experiments for $10-15 \mathrm{~min}$

${ }^{a} f \mathrm{O}_{2}$ with respect to the iron-wüstite (IW) buffer calculated assuming non-ideal solution behavior of metal and silicate melts where activity coefficient of iron in metallic melt was calculated using mole fraction of carbon, $X_{\mathrm{C}}$ calculated using the computed carbon solubility after the thermodynamic approach of Wood (1993) and detailed in Wade et al. (2012).

${ }^{b} \mathrm{fO}_{2}$ with respect to the IW buffer calculated assuming non-ideal solution of $\mathrm{Fe}$ in metal and $\mathrm{FeO}$ in silicate melts with activity coefficient of Fe in metallic melt calculated using $X_{\mathrm{C}}$ based on measured carbon content of metal melt (see text for details). This column reports our preferred oxygen fugacity values.

${ }^{c} f \mathrm{O}_{2}$ with respect to the iron-wüstite (IW) buffer calculated assuming ideal solution behavior of $\mathrm{Fe}$ in metallic and FeO in silicate melts and using the measured carbon content.

d 'Glass' denotes that the silicate melt quenched entirely or dominantly to a glassy pool. 'Dendritic' denotes that the entire melt pool is composed of dendritic quenched crystals. 
coefficients of $\mathrm{Fe}$ in Fe-rich metallic melt, $\gamma_{\mathrm{Fe}}$, were calculated using the $\varepsilon$-approach (Wagner, 1952; Ma, 2001), which takes into account the non-ideal interactions between all the components in the Fe-rich liquid and especially $\mathrm{C}$ and $\mathrm{S}$ (where present). $\gamma_{\mathrm{Fe}}$ calculations for experiments in graphite capsule employed carbon mole fraction, $X_{\mathrm{C}}$ both as measured by EPMA or SIMS and those computed by thermodynamic approach described in Wade et al. (2012) and calculated with their metal activity calculator. It is observed that the difference in calculated $f \mathrm{O}_{2}$ in these two approaches is no more than $\sim 0.1-0.2 \log$ units, with calculations that use measured $\mathrm{C}$ predicting slightly more reduced conditions (Table 2). Also reported in Table 2 are the experimental $f \mathrm{O}_{2} \mathrm{~S}$ for an ideal solution behavior of $\mathrm{FeO}$ in silicate melt and $\mathrm{Fe}$ in metallic melt, i.e., $a_{\mathrm{FeO}}$ (activity of $\mathrm{FeO})=X_{\mathrm{FeO}}($ mole fraction of $\mathrm{FeO})$ and $a_{\mathrm{Fe}}$ (activity of $\mathrm{Fe})=X_{\mathrm{Fe}}$ (mole fraction of $\mathrm{Fe}$ ).

Although hydrogen fugacity, $\mathrm{fH}_{2}$ of our experiments were not buffered, relatively narrow and appropriate range of $f \mathrm{O}_{2}$ (Table 2) along with similar range of 'water' content in our pre-dried basaltic glasses suggests that $f \mathrm{H}_{2}$ of most our experiments also varied within an appropriate range according to the following equilibrium:

$\mathrm{H}_{2}+0.5 \mathrm{O}_{2}=\mathrm{H}_{2} \mathrm{O}$

\subsubsection{High pressure-temperature experiments}

The experiments were performed at $1600^{\circ} \mathrm{C}$ and 1 and $3 \mathrm{GPa}$ (silicate compositions ThB and Knippa and metal composition 'core 3') using end-loaded piston cylinder (PC) device at Rice University and at $2000{ }^{\circ} \mathrm{C}$ and 2-5 GPa (silicate compositions MAR, MT-7C, and KLB1 ; with only one experiment performed at $2100{ }^{\circ} \mathrm{C}$ ) using a Walker-style multi anvil (MA) device at Columbia University (Table 2). The PC and MA experiments used assembly and procedure that are detailed in Tsuno and Dasgupta (2011) and Dasgupta et al. (2009), respectively. The run durations varied from $2 \mathrm{~h}$ at $1600^{\circ} \mathrm{C}$ to $10-15 \mathrm{~min}$ at 2000-2100 ${ }^{\circ} \mathrm{C}$. All the experiments were pressurized at room temperatures and held at $800{ }^{\circ} \mathrm{C}$ for $15-24 \mathrm{~h}$ for MA and $900{ }^{\circ} \mathrm{C}$ for $1-3 \mathrm{~h}$ for $\mathrm{PC}$ to minimize porosity in the capsules before the formation of metallic melts. After sintering, the experiments were heated at an average rate of $100{ }^{\circ} \mathrm{C} / \mathrm{min}-$ ute to the nominal temperature of the experiment. Experiments were terminated by cutting off power to the heater (graphite for $\mathrm{PC}$ and $\mathrm{LaCrO}_{3}$ for MA runs). The pressure uncertainty in PC and MA experiments are approximately 0.1 and $0.3 \mathrm{GPa}$, respectively. The recovered capsules were mounted in Crystal Bond ${ }^{\circledR}$ and ground longitudinally to expose approximately the medial section of the sample. All the samples were polished with 0.3 micron alumina slurry on a lapidary wheel. Typically, the samples were first carbon coated for major element analysis of metals and silicates by electron microprobe, followed by removal of carbon coating and re-coating ( $\sim 25-30 \mathrm{~nm}$ thick) with Al for carbon analysis in metallic melts by electron microprobe. Finally, Al-coats were removed, crystal bond dissolved, and the samples were mounted in Indium and gold-coated for carbon concentration determination using SIMS. Uncoated polished samples were used for Raman spectroscopy.

\subsection{Analytical techniques}

\subsubsection{Secondary ionization mass spectrometry (SIMS)}

Carbon content of experimental phases was determined using a Cameca IMS 1280, a large-radius ion microprobe, at Woods Hole Oceanographic Institution in three analytical sessions. A primary ion beam of $\mathrm{Cs}^{+}$with kinetic energy of $10 \mathrm{keV}$ and a current of $1.2-1.5 \mathrm{nA}$ was focused into a 10 micron diameter spot and was rastered over a $30 \times 30$ $50 \times 50$ square micron spot and sputtered secondary ions from the solid specimen surface were accelerated to 10-keV energy into a Nier-Johnson type double-focusing mass spectrometer and mass analyzed. To avoid surface contamination, ions were collected from the central $15 \times 15$ square micron area. Mass-analyzed ions were detected by a 22-stage Allen type electron multiplier and are digitally recorded. For quenched silicate melts, with dendritic quenched crystals, or silicate glasses, ${ }^{12} \mathrm{C} /{ }^{30} \mathrm{Si}$ intensity ratio was used as a proxy for carbon concentration. Secondary ion intensities of ${ }^{32} \mathrm{~S}$ relative to ${ }^{30} \mathrm{Si}$ were
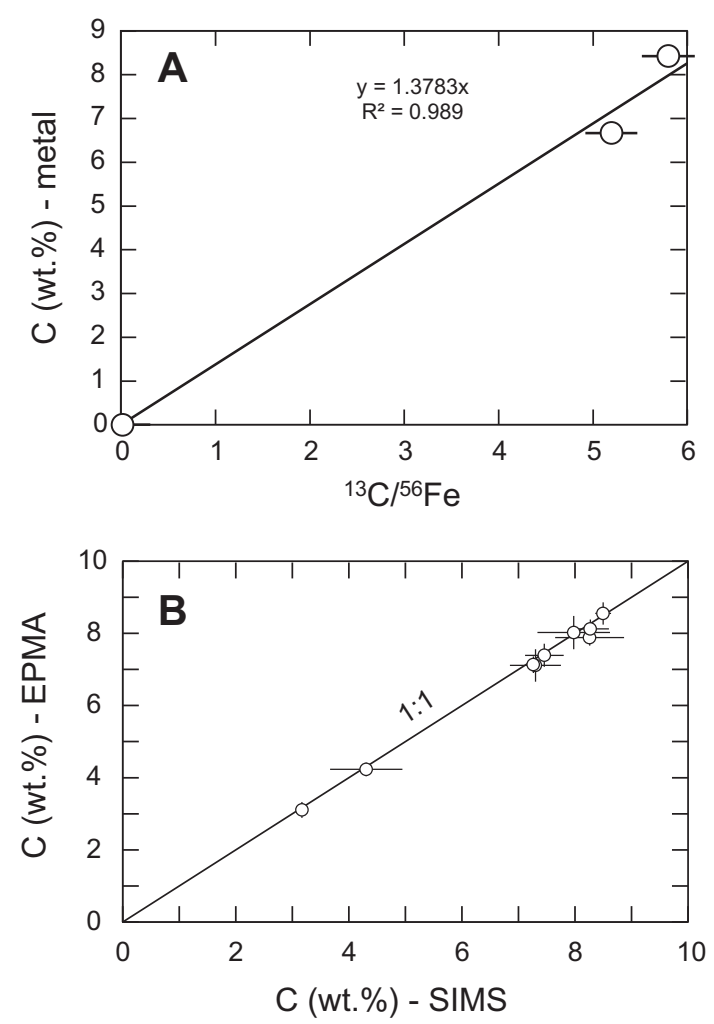

Fig. 1. (A) SIMS calibration for carbon measurement in Fe-rich metallic melts where ${ }^{13} \mathrm{C}$ ion intensity with respect to ${ }^{56} \mathrm{Fe}$ is used as a proxy for absolute carbon content in the metallic melts. Standards with known carbon concentrations included stoichiometric $\mathrm{Fe}_{3} \mathrm{C}, \mathrm{Fe}_{7} \mathrm{C}_{3}$, and nominally carbon free, reagent grade $\mathrm{Fe}$ metal (Alfa Aesar 99.995 wt.\% Fe). (B) Carbon analysis of quenched Fe-rich metallic melt for a subset of our experiments, showing good agreement between the two analytical methods SIMS and EPMA - employed. The $1 \sigma$ error for EPMA analyses does not include uncertainties owing to carbon blank values and only reflect heterogeneity based on replicate analyses. 
Table 3

\begin{tabular}{|c|c|c|c|c|c|c|c|c|c|c|c|c|c|c|c|c|c|c|}
\hline Run No. & B109 & & G212 & & B172 & & $\mathrm{B} 170$ & & B149 & & B148 & & BB-932 & & BB-929 & & BB-930 & \\
\hline $\begin{array}{l}\text { Silicate melts } \\
n\end{array}$ & $10(3)$ & & $10(2)$ & & $12(3)$ & & $11(3)$ & & $10(2)$ & & $11(2)$ & & $9(3)$ & & $11(3)$ & & $12(3)$ & \\
\hline & Average & $1 \sigma$ & Average & $1 \sigma$ & Average & $1 \sigma$ & Average & $1 \sigma$ & Average & $1 \sigma$ & Average & $1 \sigma$ & Average & $1 \sigma$ & Average & $1 \sigma$ & Average & $1 \sigma$ \\
\hline $\mathrm{SiO}_{2}$ & 47.51 & 0.33 & 47.77 & 0.41 & 40.00 & 0.50 & 40.65 & 0.35 & 38.47 & 0.33 & 39.68 & 0.69 & 49.64 & 0.54 & 49.23 & 0.44 & 49.38 & 0.38 \\
\hline $\mathrm{TiO}_{2}$ & 0.77 & 0.09 & 0.80 & 0.11 & 3.33 & 0.05 & 3.40 & 0.09 & 3.09 & 0.05 & 3.38 & 0.05 & 1.21 & 0.09 & 1.24 & 0.06 & 1.25 & 0.08 \\
\hline $\mathrm{Al}_{2} \mathrm{O}_{3}$ & 15.96 & 0.19 & 16.05 & 0.11 & 10.39 & 0.09 & 10.45 & 0.17 & 10.63 & 0.08 & 10.45 & 0.19 & 14.29 & 0.11 & 14.47 & 0.11 & 14.50 & 0.21 \\
\hline $\mathrm{Cr}_{2} \mathrm{O}_{3}$ & 0.03 & 0.01 & 0.02 & 0.01 & 0.05 & 0.02 & 0.08 & 0.02 & 0.04 & 0.01 & 0.06 & 0.02 & 0.05 & 0.01 & 0.04 & 0.02 & 0.04 & 0.01 \\
\hline $\mathrm{FeO}^{*}$ & 14.28 & 0.21 & 14.20 & 0.18 & 14.67 & 0.14 & 15.11 & 0.20 & 15.38 & 0.19 & 13.46 & 0.32 & 11.69 & 0.24 & 11.81 & 0.19 & 11.74 & 0.18 \\
\hline $\mathrm{MnO}$ & 0.20 & 0.02 & 0.20 & 0.04 & 0.20 & 0.03 & 0.22 & 0.03 & 0.16 & 0.01 & 0.20 & 0.04 & 0.17 & 0.03 & 0.17 & 0.02 & 0.15 & 0.02 \\
\hline $\mathrm{MgO}$ & 8.06 & 0.12 & 8.22 & 0.11 & 12.94 & 0.20 & 12.26 & 0.13 & 13.57 & 0.42 & 13.57 & 0.37 & 8.61 & 0.15 & 8.70 & 0.11 & 8.62 & 0.11 \\
\hline $\mathrm{NiO}$ & 0.015 & 0.003 & 0.017 & 0.004 & 0.016 & 0.003 & 0.021 & 0.005 & 0.015 & 0.006 & 0.019 & 0.007 & 0.025 & 0.006 & 0.037 & 0.004 & 0.048 & 0.010 \\
\hline $\mathrm{CoO}$ & - & & - & & - & & - & & - & & - & & 0.006 & 0.002 & 0.004 & 0.002 & 0.008 & 0.004 \\
\hline $\mathrm{CaO}$ & 10.19 & 0.11 & 10.19 & 0.08 & 12.14 & 0.10 & 11.71 & 0.07 & 11.87 & 0.09 & 12.30 & 0.17 & 11.00 & 0.15 & 11.20 & 0.14 & 11.30 & 0.11 \\
\hline $\mathrm{Na}_{2} \mathrm{O}$ & 2.64 & 0.05 & 2.59 & 0.06 & 3.22 & 0.07 & 3.01 & 0.07 & 2.85 & 0.01 & 2.41 & 0.15 & 2.22 & 0.11 & 2.24 & 0.08 & 2.28 & 0.09 \\
\hline $\mathrm{K}_{2} \mathrm{O}$ & 0.09 & 0.02 & 0.10 & 0.03 & 1.78 & 0.03 & 1.69 & 0.05 & 1.68 & 0.00 & 1.81 & 0.07 & 0.08 & 0.01 & 0.08 & 0.01 & 0.08 & 0.01 \\
\hline $\mathrm{P}_{2} \mathrm{O}_{5}$ & - & & - & & 0.67 & 0.06 & 0.63 & 0.06 & 0.63 & 0.05 & 1.00 & 0.32 & & & & & & \\
\hline S (EPMA) & & & & & & & & & & & & & 0.11 & 0.013 & 0.08 & 0.013 & 0.078 & 0.01 \\
\hline Sum & 99.74 & & 100.16 & & 99.40 & & 99.21 & & 98.38 & & 98.33 & & 99.10 & & 99.31 & & 99.47 & \\
\hline $\mathrm{C}(\mathrm{SIMS}-\mathrm{ppm})$ & 24 & 1 & 12 & 1 & 74 & 4 & 39 & 2 & 108 & 1 & 58 & 2 & 55 & 2 & 24 & 1 & 19 & 2 \\
\hline S (SIMS) & & & & & & & & & & & & & 0.109 & 0.008 & 0.0642 & 0.00083 & 0.049 & 0.001 \\
\hline $\mathrm{H}_{2} \mathrm{O}$ (SIMS) & 0.557 & 0.024 & 0.339 & 0.001 & 0.289 & 0.011 & 0.415 & 0.005 & 1.761 & 0.004 & 1.887 & 0.005 & 0.57 & 0.03 & 0.51 & 0.01 & 0.672 & 0.002 \\
\hline \multirow{3}{*}{$\begin{array}{l}\text { Metallic melts } \\
n\end{array}$} & & & & & & & & & & & & & & & & & & \\
\hline & 8 & & 7 & & 9 & & 11 & & 10 & & 3 & & 8 & & 9 & & 7 & \\
\hline & Average & $1 \sigma$ & Average & $1 \sigma$ & Average & $1 \sigma$ & Average & $1 \sigma$ & Average & $1 \sigma$ & Average & $1 \sigma$ & Average & $1 \sigma$ & Average & $1 \sigma$ & Average & $1 \sigma$ \\
\hline $\mathrm{Cr}$ & & & & & & & & & - & & - & & & & & & & \\
\hline $\mathrm{Fe}$ & 88.97 & 0.23 & 88.34 & 0.37 & 89.02 & 0.42 & 89.05 & 0.32 & 88.94 & 0.35 & 88.62 & 0.48 & 85.29 & 0.32 & 85.83 & 0.12 & 84.63 & 0.92 \\
\hline $\mathrm{Mn}$ & - & & - & & - & & - & & - & & - & & - & - & - & - & - & - \\
\hline $\mathrm{Ni}$ & 6.25 & 0.70 & 6.03 & 0.23 & 5.74 & 0.09 & 5.72 & 0.06 & 4.85 & 0.09 & 4.80 & 0.09 & 7.29 & 0.25 & 6.70 & 0.14 & 6.79 & 0.12 \\
\hline Co & - & & - & & - & & - & & - & & - & & 0.23 & 0.04 & 0.21 & 0.03 & 0.24 & 0.05 \\
\hline C (EPMA) & 4.84 & 0.48 & 5.61 & 0.18 & 5.00 & 0.20 & 5.35 & 0.26 & 6.07 & 0.21 & 6.83 & 0.72 & 5.80 & 0.24 & 5.84 & 0.41 & 6.82 & 0.28 \\
\hline S (EPMA) & - & & - & & - & & - & & - & & - & & 1.52 & 0.13 & 1.53 & 0.17 & 1.66 & 0.17 \\
\hline Sum & 100.06 & 0.28 & 99.98 & 0.23 & 99.76 & 0.46 & 100.12 & 0.32 & 99.85 & 0.31 & 100.25 & 0.54 & 100.14 & & 100.11 & & 100.14 & \\
\hline C (SIMS) & - & & - & & - & & - & & - & & - & & - & & - & & - & \\
\hline$D_{\mathrm{C}}($ met-sil) & 2060 & 231 & 4757 & 355 & 676 & 43 & 1390 & 98 & 563 & 20 & 1169 & 130 & 1056 & 58 & 2457 & 201 & 3639 & 416 \\
\hline$D_{\mathrm{S}}($ met-sil) & & & & & & & & & & & & & 13.8 & 2.0 & 19.1 & 3.7 & 21.3 & 3.5 \\
\hline$D_{\mathrm{S}}(\text { met-sil })^{*}$ & & & & & & & & & & & & & 13.9 & 1.6 & 23.8 & 2.6 & 33.6 & 3.4 \\
\hline$D_{\mathrm{Ni}}($ met-sil) & 530 & 121 & 451 & 108 & 457 & 86 & 347 & 83 & 411 & 165 & 321 & 119 & 364 & 89 & 233 & 27 & 179 & 37 \\
\hline$D_{\mathrm{Co}}($ met-sil) & & & & & & & & & & & & & 51 & 22 & 62 & 34 & 37 & 20 \\
\hline
\end{tabular}




\begin{tabular}{|c|c|c|c|c|c|c|c|c|c|c|c|c|c|c|c|c|c|c|c|c|c|c|}
\hline Run No. & BB-914 & & BB-905 & & BB-904 & & BB-901 & & BB-919 & & BB-920 & & BB-916 & & BB-918 & & BB-921 & & BB-940 & & B-939 & \\
\hline $\begin{array}{l}\text { Silicate melts } \\
n\end{array}$ & $\begin{array}{l}12(7) \\
\text { Average }\end{array}$ & $1 \sigma$ & $\begin{array}{l}17(6) \\
\text { Average }\end{array}$ & $1 \sigma$ & $\begin{array}{l}20(5) \\
\text { Average }\end{array}$ & $1 \sigma$ & $\begin{array}{l}16(7) \\
\text { Average }\end{array}$ & $1 \sigma$ & $\begin{array}{l}29(5) \\
\text { Average }\end{array}$ & $1 \sigma$ & $\begin{array}{l}23(6) \\
\text { Average }\end{array}$ & $1 \sigma$ & $\begin{array}{l}24(5) \\
\text { Average }\end{array}$ & $1 \sigma$ & $\begin{array}{l}27(4) \\
\text { Average }\end{array}$ & $1 \sigma$ & $\begin{array}{l}19(5) \\
\text { Average }\end{array}$ & $1 \sigma$ & $\begin{array}{l}21(2) \\
\text { Average }\end{array}$ & $1 \sigma$ & $\begin{array}{l}21(1) \\
\text { Average }\end{array}$ & $1 \sigma$ \\
\hline $\begin{array}{l}\mathrm{SiO}_{2} \\
\mathrm{TiO}_{2} \\
\mathrm{Al}_{2} \mathrm{O}_{3} \\
\mathrm{Cr}_{2} \mathrm{O}_{3} \\
\mathrm{FeO}^{*} \\
\mathrm{MnO} \\
\mathrm{MgO} \\
\mathrm{NiO} \\
\mathrm{CoO} \\
\mathrm{CaO} \\
\mathrm{Na}_{2} \mathrm{O} \\
\mathrm{K}_{2} \mathrm{O}\end{array}$ & $\begin{array}{l}45.35 \\
0.37 \\
7.46 \\
0.37 \\
12.25 \\
0.19 \\
25.55 \\
0.014 \\
0.057 \\
7.11 \\
0.55 \\
0.13\end{array}$ & $\begin{array}{l}0.61 \\
0.01 \\
0.16 \\
0.04 \\
0.08 \\
0.01 \\
0.52 \\
0.005 \\
0.015 \\
0.12 \\
0.02 \\
0.01\end{array}$ & $\begin{array}{l}40.11 \\
0.31 \\
6.56 \\
0.27 \\
11.37 \\
0.15 \\
33.89 \\
0.026 \\
0.064 \\
6.56 \\
0.41 \\
0.08\end{array}$ & $\begin{array}{l}0.64 \\
0.09 \\
0.42 \\
0.03 \\
0.49 \\
0.05 \\
1.08 \\
0.010 \\
0.021 \\
0.52 \\
0.04 \\
0.04\end{array}$ & $\begin{array}{l}40.13 \\
0.29 \\
6.70 \\
0.27 \\
11.56 \\
0.15 \\
33.94 \\
0.033 \\
0.075 \\
6.68\end{array}$ & $\begin{array}{l}0.76 \\
0.09 \\
0.53 \\
0.04 \\
0.59 \\
0.03 \\
1.75 \\
0.012 \\
0.023 \\
0.71 \\
0.13 \\
0.03\end{array}$ & $\begin{array}{l}44.60 \\
0.36 \\
7.49 \\
0.30 \\
12.12 \\
0.18 \\
26.21 \\
0.025 \\
0.084 \\
7.23 \\
0.59 \\
0.12\end{array}$ & $\begin{array}{l}0.89 \\
0.13 \\
0.41 \\
0.03 \\
0.57 \\
0.03 \\
0.60 \\
0.005 \\
0.009 \\
0.35 \\
0.18 \\
0.02\end{array}$ & $\begin{array}{l}38.38 \\
0.41 \\
6.32 \\
0.23 \\
13.09 \\
0.16 \\
34.79 \\
0.037 \\
0.064 \\
5.29 \\
0.48 \\
0.04\end{array}$ & $\begin{array}{l}0.91 \\
0.04 \\
0.59 \\
0.05 \\
0.96 \\
0.01 \\
1.01 \\
0.011 \\
0.014 \\
0.71 \\
0.03 \\
0.02\end{array}$ & $\begin{array}{l}44.41 \\
0.31 \\
4.12 \\
0.18 \\
11.12 \\
0.16 \\
35.31 \\
0.044 \\
0.076 \\
3.86 \\
0.36 \\
0.02\end{array}$ & $\begin{array}{l}1.03 \\
0.03 \\
0.71 \\
0.10 \\
0.81 \\
0.01 \\
1.71 \\
0.010 \\
0.031 \\
0.50 \\
0.03 \\
0.01\end{array}$ & $\begin{array}{l}46.03 \\
0.44 \\
5.79 \\
0.16 \\
11.98 \\
0.18 \\
28.87 \\
0.041 \\
0.104 \\
5.67 \\
0.56 \\
0.02\end{array}$ & $\begin{array}{l}0.88 \\
0.13 \\
0.74 \\
0.07 \\
0.51 \\
0.06 \\
1.58 \\
0.011 \\
0.038 \\
0.57 \\
0.13 \\
0.02\end{array}$ & $\begin{array}{l}34.33 \\
0.37 \\
6.85 \\
0.25 \\
10.60 \\
0.12 \\
38.62 \\
0.051 \\
0.095 \\
7.54\end{array}$ & $\begin{array}{l}1.32 \\
0.08 \\
1.59 \\
0.09 \\
1.81 \\
0.02 \\
3.68 \\
0.012 \\
0.014 \\
1.92 \\
0.15 \\
0.09\end{array}$ & $\begin{array}{l}33.79 \\
0.27 \\
6.12 \\
0.34 \\
10.56 \\
0.12 \\
40.88 \\
0.037 \\
0.102 \\
6.68 \\
0.48 \\
0.13\end{array}$ & $\begin{array}{l}1.00 \\
0.05 \\
0.55 \\
0.06 \\
0.56 \\
0.01 \\
0.95 \\
0.012 \\
0.008 \\
0.46 \\
0.02 \\
0.01\end{array}$ & $\begin{array}{l}33.81 \\
1.11 \\
11.02 \\
0.07 \\
8.85 \\
0.13 \\
31.88 \\
0.046 \\
0.007 \\
11.35 \\
2.03 \\
0.09\end{array}$ & $\begin{array}{l}0.92 \\
0.34 \\
0.49 \\
0.04 \\
0.85 \\
0.03 \\
0.91 \\
0.009 \\
0.002 \\
0.31 \\
0.11 \\
0.02\end{array}$ & $\begin{array}{l}37.37 \\
1.31 \\
9.29 \\
0.03 \\
8.97 \\
0.11 \\
27.37 \\
0.052 \\
0.008 \\
13.55 \\
2.05 \\
0.15\end{array}$ & $\begin{array}{l}0.81 \\
0.13 \\
0.66 \\
0.01 \\
0.21 \\
0.03 \\
0.45 \\
0.009 \\
0.002 \\
0.54 \\
0.12 \\
0.03\end{array}$ \\
\hline $\begin{array}{l}\text { S (EPMA) } \\
\text { Sum }\end{array}$ & $\begin{array}{l}0.26 \\
99.67\end{array}$ & 0.02 & $\begin{array}{l}0.11 \\
99.95\end{array}$ & 0.02 & $\begin{array}{l}0.16 \\
100.53\end{array}$ & 0.03 & $\begin{array}{l}0.04 \\
99.41\end{array}$ & 0.01 & $\begin{array}{l}0.17 \\
99.54\end{array}$ & 0.04 & $\begin{array}{l}0.11 \\
100.10\end{array}$ & 0.03 & $\begin{array}{l}0.09 \\
99.96\end{array}$ & 0.02 & $\begin{array}{l}0.08 \\
99.55\end{array}$ & 0.02 & $\begin{array}{l}0.10 \\
99.61\end{array}$ & 0.02 & $\begin{array}{l}0.10 \\
100.49\end{array}$ & 0.03 & $\begin{array}{l}0.10 \\
100.37\end{array}$ & 0.02 \\
\hline $\begin{array}{l}\mathrm{C}(\mathrm{SIMS}-\mathrm{ppm}) \\
\mathrm{S}(\mathrm{SIMS}) \\
\mathrm{H}_{2} \mathrm{O} \text { (SIMS) }\end{array}$ & $\begin{array}{l}92 \\
0.111 \\
0.54\end{array}$ & $\begin{array}{l}12 \\
0.003 \\
0.06\end{array}$ & $\begin{array}{l}0.104 \\
0.49\end{array}$ & $\begin{array}{l}30 \\
0.007 \\
0.05\end{array}$ & $\begin{array}{l}354 \\
0.103 \\
0.61\end{array}$ & $\begin{array}{l}32 \\
0.007 \\
0.04\end{array}$ & $\begin{array}{l}597 \\
0.042 \\
0.62\end{array}$ & $\begin{array}{l}119 \\
0.004 \\
0.02\end{array}$ & $\begin{array}{l}0.052 \\
0.43\end{array}$ & $\begin{array}{l}100 \\
0.009 \\
0.02\end{array}$ & $\begin{array}{l}0.094 \\
0.51\end{array}$ & $\begin{array}{l}122 \\
0.008 \\
0.02\end{array}$ & $\begin{array}{l}0.036 \\
0.61\end{array}$ & $\begin{array}{l}50 \\
0.007 \\
0.04\end{array}$ & $\begin{array}{l}86 \\
0.177 \\
0.37\end{array}$ & $\begin{array}{l}0.025 \\
0.06\end{array}$ & $\begin{array}{l}174 \\
0.177 \\
0.72\end{array}$ & $\begin{array}{l}76 \\
0.005 \\
0.03\end{array}$ & $\begin{array}{l}112 \\
0.05 \\
0.65\end{array}$ & $\begin{array}{l}21 \\
0.01 \\
0.01\end{array}$ & $\begin{array}{l}325 \\
0.33 \\
0.84\end{array}$ & \\
\hline $\begin{array}{l}\text { Metallic melts } \\
n\end{array}$ & $\begin{array}{l}10(6) \\
\text { Average }\end{array}$ & $1 \sigma$ & $\begin{array}{l}7(5) \\
\text { Average }\end{array}$ & $1 \sigma$ & $\begin{array}{l}8(7) \\
\text { Average }\end{array}$ & $1 \sigma$ & $\begin{array}{l}7 \text { (6) } \\
\text { Average }\end{array}$ & $1 \sigma$ & $\begin{array}{l}11(5) \\
\text { Average }\end{array}$ & $1 \sigma$ & $\begin{array}{l}9(6) \\
\text { Average }\end{array}$ & $1 \sigma$ & $\begin{array}{l}13(5) \\
\text { Average }\end{array}$ & $1 \sigma$ & $\begin{array}{l}8(7) \\
\text { Average }\end{array}$ & $1 \sigma$ & $\begin{array}{l}6(6) \\
\text { Average }\end{array}$ & & $\begin{array}{l}7 \\
\text { Average }\end{array}$ & $1 \sigma$ & $\begin{array}{l}8 \\
\text { Average }\end{array}$ & $1 \sigma$ \\
\hline $\begin{array}{l}\mathrm{Cr} \\
\mathrm{Fe} \\
\mathrm{Mn} \\
\mathrm{Ni} \\
\mathrm{Co} \\
\mathrm{C} \text { (EPMA) } \\
\mathrm{S} \text { (EPMA) } \\
\text { Sum }\end{array}$ & $\begin{array}{l}0.12 \\
84.56 \\
0.00 \\
3.15 \\
3.41 \\
7.05 \\
1.35 \\
99.64\end{array}$ & $\begin{array}{l}0.04 \\
0.80 \\
0.00 \\
0.02 \\
0.16 \\
0.26 \\
0.02\end{array}$ & $\begin{array}{l}- \\
83.01 \\
- \\
5.11 \\
3.18 \\
7.13 \\
1.71 \\
100.14\end{array}$ & $\begin{array}{l}0.06 \\
0.11 \\
0.23 \\
0.13\end{array}$ & $\begin{array}{l}- \\
82.32 \\
- \\
5.04 \\
3.57 \\
7.59 \\
1.68 \\
100.20\end{array}$ & $\begin{array}{l}0.12 \\
0.08 \\
0.32 \\
0.10\end{array}$ & $\begin{array}{l}- \\
83.27 \\
- \\
3.91 \\
3.00 \\
8.02 \\
1.88 \\
100.07\end{array}$ & $\begin{array}{l}0.09 \\
0.09 \\
0.46 \\
0.12\end{array}$ & $\begin{array}{l}0.11 \\
81.26 \\
0.01 \\
5.16 \\
4.04 \\
7.88 \\
1.43 \\
99.89\end{array}$ & $\begin{array}{l}0.04 \\
0.51 \\
0.01 \\
0.04 \\
0.09 \\
0.22 \\
0.10\end{array}$ & $\begin{array}{l}0.15 \\
81.81 \\
0.01 \\
5.13 \\
3.52 \\
7.51 \\
1.84 \\
99.97\end{array}$ & $\begin{array}{l}0.11 \\
0.76 \\
0.01 \\
0.16 \\
0.19 \\
0.45 \\
0.08\end{array}$ & $\begin{array}{l}81.61 \\
0.02 \\
3.96 \\
4.04 \\
8.55 \\
1.75 \\
99.99\end{array}$ & $\begin{array}{l}0.04 \\
0.51 \\
0.01 \\
0.04 \\
0.09 \\
0.31 \\
0.10\end{array}$ & $\begin{array}{l}0.03 \\
83.22 \\
0.01 \\
6.74 \\
4.10 \\
4.23 \\
1.67 \\
100.00\end{array}$ & $\begin{array}{l}0.03 \\
0.07 \\
0.01 \\
0.14 \\
0.09 \\
0.19 \\
0.08\end{array}$ & $\begin{array}{l}0.06 \\
84.73 \\
0.01 \\
6.35 \\
4.23 \\
3.11 \\
1.63 \\
100.11\end{array}$ & $\begin{array}{l}0.053 \\
0.656 \\
0.009 \\
0.036 \\
0.288 \\
0.22 \\
0.165\end{array}$ & $\begin{array}{l}88.05 \\
- \\
8.09 \\
0.21 \\
1.78 \\
2.08 \\
100.21\end{array}$ & $\begin{array}{l}1.791 \\
- \\
0.301 \\
0.03 \\
0.039 \\
0.31\end{array}$ & $\begin{array}{l}88.25 \\
- \\
8.31 \\
0.23 \\
1.57 \\
1.76 \\
100.13\end{array}$ & $\begin{array}{l}1.068 \\
- \\
0.237 \\
0.05 \\
0.051 \\
0.21\end{array}$ \\
\hline C (SIMS) & 1.19 & 0.33 & 7.26 & 0.12 & 7.56 & 0.34 & 1.98 & 0.64 & 8.20 & 0.61 & 7.40 & 0.45 & 8.50 & 0.14 & 4.31 & 0.64 & 3.17 & 0.06 & - & - & - & - \\
\hline $\begin{array}{l}D_{\mathrm{C}}(\text { met-sil }) \\
D_{\mathrm{S}}(\text { met-sil }) \\
D_{\mathrm{S}}\left(\text { met-sil }{ }^{*}\right. \\
D_{\mathrm{Ni}} \text { (met-sil) } \\
D_{\mathrm{Co}} \text { (met-sil) }\end{array}$ & $\begin{array}{l}766 \\
5.3 \\
12.1 \\
286 \\
76\end{array}$ & $\begin{array}{l}104 \\
0.3 \\
0.4 \\
102 \\
20\end{array}$ & $\begin{array}{l}239 \\
15.5 \\
16.4 \\
250 \\
63\end{array}$ & $\begin{array}{l}25 \\
3.1 \\
1.7 \\
96 \\
21\end{array}$ & $\begin{array}{l}214 \\
10.5 \\
16.3 \\
193 \\
61\end{array}$ & $\begin{array}{l}21 \\
2.1 \\
1.5 \\
67 \\
19\end{array}$ & $\begin{array}{l}134 \\
46.9 \\
44.6 \\
199 \\
45\end{array}$ & $\begin{array}{l}28 \\
12.1 \\
5.1 \\
36 \\
5\end{array}$ & $\begin{array}{l}8.4 \\
27.5 \\
177 \\
80\end{array}$ & $\begin{array}{l}16 \\
2.0 \\
5.1 \\
54 \\
18\end{array}$ & $\begin{array}{l}16.7 \\
19.5 \\
147 \\
59\end{array}$ & $\begin{array}{l}143 \\
4.6 \\
1.8 \\
35 \\
25\end{array}$ & $\begin{array}{l}19.5 \\
48.1 \\
123 \\
49\end{array}$ & $\begin{array}{l}89 \\
4.2 \\
9.6 \\
35 \\
18\end{array}$ & $\begin{array}{l}20.9 \\
9.4 \\
169 \\
55\end{array}$ & $\begin{array}{l}55 \\
5.3 \\
1.4 \\
40 \\
8\end{array}$ & $\begin{array}{l}179 \\
15.6 \\
9.2 \\
217 \\
53\end{array}$ & $\begin{array}{l}79 \\
3.4 \\
1.0 \\
73 \\
6\end{array}$ & $\begin{array}{l}159 \\
20.8 \\
42.0 \\
224 \\
38\end{array}$ & $\begin{array}{l}30 \\
7.0 \\
10.6 \\
45 \\
12\end{array}$ & $\begin{array}{l}48 \\
17.4 \\
5.4 \\
203 \\
37\end{array}$ & $\begin{array}{l}2 \\
4.0 \\
0.6 \\
36 \\
12\end{array}$ \\
\hline
\end{tabular}

$n$ - number of analyses averaged to obtain the composition reported; 10 (3) should be read as 10 spot analyses using EPMA and 3 spot analyses using SIMS. All the numbers are in wt.\% unless otherwise stated. $D_{\mathrm{C}}$ (met-sil) is the partition coefficient of carbon between metallic and silicate melt and derived by dividing the concentration of carbon (by weight) measured in metallic melt using EPMA by the concentration of carbon (by weight) in silicate melt measured using SIMS. No significant difference in the estimated $D_{\mathrm{C}}$ (met-sil) is noticed if SIMS determined C contents of metallic melts are used. All the other $D$ (met-sil) are calculated using elemental concentrations determined using EPMA except in $D_{\mathrm{S}}$ (met-sil) ${ }^{*}$, where $\mathrm{S}$ contents determined using SIMS are used.

'-, sign indicates that the element or oxide in question was not measured. 
also measured in a subset of samples in order to verify EPMA-measured silicate melt $\mathrm{S}$ content. Water content of silicates was measured using ${ }^{1} \mathrm{H}^{16} \mathrm{O} /{ }^{30} \mathrm{Si}$ as the proxy. We also measured ${ }^{13} \mathrm{C} /{ }^{56} \mathrm{Fe}$ intensity ratios in some of our metallic melts to determine carbon content of the same. We chose to measure ${ }^{13} \mathrm{C}$ instead of more abundant ${ }^{12} \mathrm{C}$ because both our analytical standards and samples gave too high ion intensities with the use of the latter, causing saturation of electron multiplier. For each spot, 6-10 cycles of ion intensities were measured. If the ion intensities did not remain stable through the cycles, owing likely to contamination, the selected spot analysis was treated as contaminated and rejected.

Linear calibration curves for carbon content in silicate and metallic melt as a function of ${ }^{12} \mathrm{C} /{ }^{30} \mathrm{Si}$ and ${ }^{13} \mathrm{C} /{ }^{56} \mathrm{Fe}$ intensity ratios respectively were obtained in every analytical session. Analytical standards for carbon and water in silicate glasses were $4-5$ basaltic glasses with known contents of $\mathrm{CO}_{2}$ and $\mathrm{H}_{2} \mathrm{O}$ (characterized by FTIR spectroscopy or manometry) in the range $\sim 50-3000 \mathrm{ppm}$ and $0.1-$ 1.0 wt. $\%$, respectively (e.g., Helo et al., 2011). Calibration curve for Fe-rich metallic melt analysis employed laboratory synthesized, stoichiometric $\mathrm{Fe}_{3} \mathrm{C} \quad(6.68 \mathrm{wt} . \% \quad \mathrm{C})$, $\mathrm{Fe}_{7} \mathrm{C}_{3}(8.4 \mathrm{wt} . \%)$ and reagent grade metallic $\mathrm{Fe}(0 \mathrm{wt} . \%$ C) (Fig. 1A).

\subsubsection{Electron probe microanalysis (EPMA)}

Major element compositions of coexisting silicate and metallic melts were determined using the Cameca SX 100 electron microprobe at the American Museum of Natural History (AMNH) and NASA-Johnson Space Center (NASA-JSC). Finely polished experimental samples were analyzed with both carbon (silicate and metallic melts without carbon as part of the analytical routine) and aluminum (carbon analyzed along with other elements in the metallic melts) as conductive coatings. Major elements in silicate ( $\mathrm{Si}, \mathrm{Ti}, \mathrm{Al}, \mathrm{Fe}, \mathrm{Mg}, \mathrm{Ca}, \mathrm{Na}, \mathrm{K}$ ) and metallic $(\mathrm{Fe}+\mathrm{Ni} \pm \mathrm{Co})$ melts were analyzed using $15 \mathrm{kV}$ accelerating voltage, $20 \mathrm{nA}$ beam current, and 10-20 s peak and background counting time. Silicate glasses were analyzed with a 10 micron diameter beam whereas metallic and silicate melts quenched to heterogeneous aggregate of dendritic crystals were analyzed with a 30 micron beam. To obtain average compositions of silicate melts that did not quench to a glass, a greater number of spot analyses was averaged (Table 3 ). For S, a peak and background counting time of $100 \mathrm{~s}$ was used. For $\mathrm{Ni}$ and $\mathrm{Co}$ in silicate melts, a beam current of $100 \mathrm{nA}$ and peak counting time of 100-200s were employed. Synthetic basaltic melts, natural minerals, and oxides were used as analytical standards at NASA-JSC whereas natural and synthetic minerals were used as analytical standards at $\mathrm{AMNH}$ for silicate melts. Pure $\mathrm{Fe}, \mathrm{Ni}$, and $\mathrm{Co}$ metal and troilite were used as standards for $\mathrm{Fe}, \mathrm{Ni}, \mathrm{Co}$, and $\mathrm{S}$, respectively for metallic melts. Carbon analysis of quenched, Fe-rich metallic melts were done following the protocol of Dasgupta and Walker (2008), using synthetic, stoichiometric $\mathrm{Fe}_{3} \mathrm{C}$ and $\mathrm{Fe}_{7} \mathrm{C}_{3}$ as primary and secondary standards, respectively. Carbon content of metallic melts measured using both EPMA and SIMS were similar within uncertainty, thus lending confidence to the estimates (Fig. 1B).

\subsubsection{Raman spectroscopy}

Samples, where glassy melt pools were obtained, were studied using Raman spectroscopy to gain insight into the possible mechanism of carbon dissolution in basaltic silicate melts at reduced, magma ocean conditions. Raman spectra were collected using a Renishaw inVia Raman microscope and a $514 \mathrm{~nm}$ laser at the Shared Equipment Authority of Rice University. Using a 50x objective lens and working power of $15.6 \mathrm{~mW}$, spectra in the frequency range of $200-4500 \mathrm{~cm}^{-1}$ were collected with $1 \mathrm{~cm}^{-1}$ resolution. A spectrum at each point was accumulated five times.

\section{RESULTS}

\subsection{Texture and phase assemblage}

All the experiments produced quenched blobs of $\mathrm{Fe}-\mathrm{Ni}$ rich metallic melt embedded in silicate melt. Experiments with composition ThB, MAR, Knippa, and Knippa-H typically produced at least partly glassy silicate matrix (3 GPa experiments with Knippa yielded some quenched crystalbearing domains along the capsule wall and/or in contact with metallic melt) whereas more depolymerized silicate melt compositions, i.e., komatiite and KLB-1 or compositions run in $\mathrm{MgO}$ capsules, which also resulted in more depolymerized silicate melt compositions, produced a dendritic quenched mass composed of long blades of olivine crystals and interstitial glassy patches (Fig. 2a). Experiments in $\mathrm{MgO}$ capsules also produced ferropericlase crystals as part of the assemblage, mostly along the capsule wall, but also dispersed within the silicate melt pool or adjacent to the metallic melt blobs. Metallic melts always quenched to an intergrowth of metallic $\mathrm{Fe}-\mathrm{Ni}$ alloy blades and Fe-rich carbides for all the runs conducted in graphite capsules. Based on texture, graphite blades when present either in metallic or silicate melt domains were treated as recrystallized equilibrium graphite rather than products of quench. Experiments that were doped with carbon below the saturation value and run in $\mathrm{MgO}$ capsules also quenched to an intergrowth of Fe-rich alloy and minor Fe-carbides, plus trace oxides in the quench aggregates (Fig. 2b).

\subsection{Major element compositions}

The experimental silicate melt compositions showed expected variations depending on the starting silicate composition and the capsule material used. The mix containing the composition MAR in a graphite capsule produced glasses with $\sim 49$ wt. $\% \quad \mathrm{SiO}_{2}, \sim 14-15$ wt. $\% \quad \mathrm{Al}_{2} \mathrm{O}_{3}$, $\sim 8.5$ wt. $\% \mathrm{MgO}$, and $11.5-12$ wt. $\% \mathrm{FeO}^{*}$ and $\mathrm{ThB}$ in a graphite capsule produced glasses with $\sim 47.5-48 \mathrm{wt} . \%$ $\mathrm{SiO}_{2}, \sim 16$ wt. $\% \mathrm{Al}_{2} \mathrm{O}_{3}, \sim 8$ wt. $\% \mathrm{MgO}$, and $\sim 14.2$ wt. $\%$ $\mathrm{FeO}^{*}$. MAR and ThB run in graphite capsules resulted in similar melt compositions and are the most polymerized of all the experiments with $\mathrm{NBO} / \mathrm{T}$ of $\sim 0.85$ (Table 3 ). The experiments using alkali basalt Knippa in a graphite 

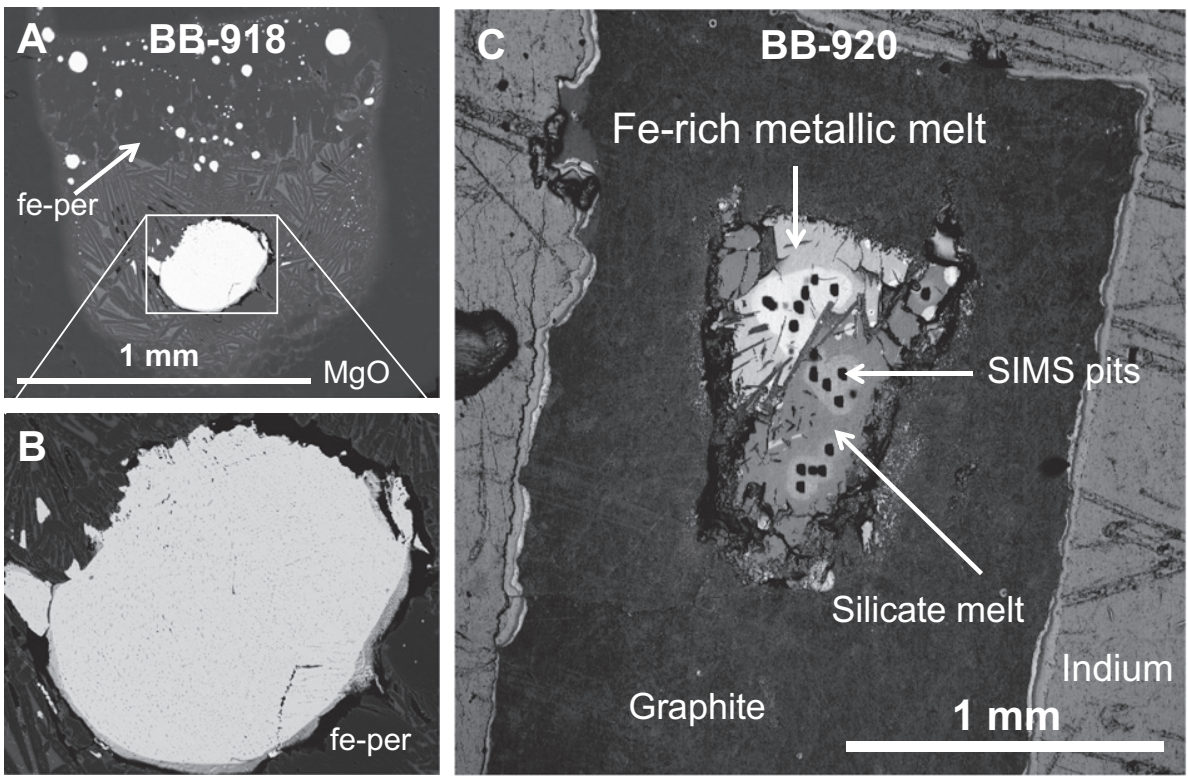

Fig. 2. Typical texture for immiscible metallic and silicate melt. (A) and (B) show quenched texture of coexisting metallic and silicate melt in an $\mathrm{MgO}$ capsule with silicate composition MT7-C (Run No. BB-918: $3 \mathrm{GPa}, 2000^{\circ} \mathrm{C}$ ). Silicate melt quenched to a dendritic network of olivine crystals with interstitial domains that appear glassy. The reaction of the capsule with the silicates also resulted in ferropericlase crystals that mostly line the capsule wall. In the experiments using $\mathrm{MgO}$ capsules, the source of carbon was $\sim 1 \mathrm{wt} . \%$ polycrystalline diamond powder added to the metal fraction (Table 1), which caused a smaller volume fraction of Fe-rich carbides (darker specs in the metal blob) in the quenched mass of metallic melt (B). (C) An experiment with KLB-1 peridotite as the silicate fraction contained in a graphite capsule (Run No. BB-920: $4 \mathrm{GPa}, 2000{ }^{\circ} \mathrm{C}$ ) showing analysis pits for SIMS in a sample mounted in In.

capsule yielded at least partly glassy quenched melt with $\sim 39$ wt. $\% \mathrm{SiO}_{2}, \sim 10.8$ wt. $\% \mathrm{Al}_{2} \mathrm{O}_{3}, 13.2-13.5$ wt. $\% \mathrm{MgO}$, and $12-15 \mathrm{wt} . \% \mathrm{FeO}^{*}$. These experiments yielded melt compositions with intermediate $\mathrm{NBO} / \mathrm{T}$ of $\sim 1.4-1.5$. Two out of four experiments with these compositions where an unfired Knippa composition was used also resulted in electron microprobe total of $97-98.5 \mathrm{wt} . \%$, suggesting the possibility of higher abundance of 'water' in these glasses. The experiments with KLB-1 and MT-7C contained in graphite capsules produced quenched melts with $39-45$ wt. $\% \mathrm{SiO}_{2}, 4.3-$ 7.5 wt. $\% \mathrm{Al}_{2} \mathrm{O}_{3}, 11-13$ wt. $\% \mathrm{FeO}^{*}$, and $26-35$ wt. $\% \mathrm{MgO}$. These melts span a range of NBO/T from 1.9 to 2.8 (Table 3). All the experiments run in $\mathrm{MgO}$ capsules, especially those with tholeitic basalt MAR as the silicate fraction, showed compositional evolution owing to reaction with $\mathrm{MgO}$. These experiments generate a magma with 33-38 wt. $\% \mathrm{SiO}_{2}, 9-13$ wt. $\% \mathrm{Al}_{2} \mathrm{O}_{3}, 8-9$ wt. $\% \mathrm{FeO}^{*}$, and $27-32 \mathrm{wt} . \% \mathrm{MgO}$.

Metallic melts in all the experiments were Fe-rich with $\mathrm{Fe}$ content varying from $\sim 81$ to $88 \mathrm{wt} . \%$. Graphite-saturated experiments with metal alloy composition 'core 1' (Table 1) produced alloy melt with $\mathrm{Ni}$ content in the range $\sim 3.2-5.2$ wt.\% whereas those runs with metal alloy 'core 2' (Table 1), produced melt with $\mathrm{Ni}$ content of $6.4-7.3 \mathrm{wt} . \%$ (Table 3), and experiments with 'core 3' (Table 1) produced metallic melts with intermediate Ni contents (4.8-6.3 wt.\%). All the metallic melts with 'core 1' and 'core 2' starting compositions were sulfur-bearing with $\mathrm{S}$ content varying from $\sim 1.3$ to $2 \mathrm{wt} . \%$, whereas experiments with 'core 3 ' were sulfur-free.

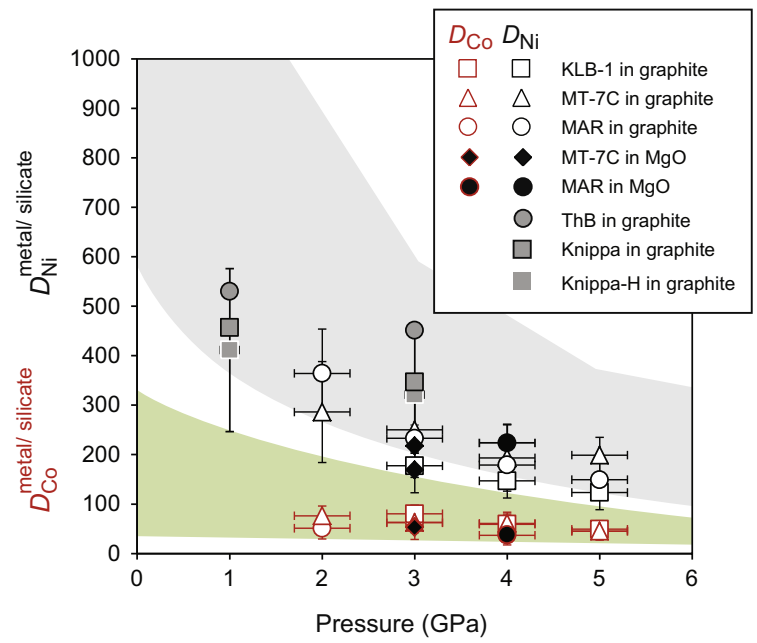

Fig. 3. Measured partition coefficients of $\mathrm{Ni}$ and Co between Ferich metallic alloy melt and mafic-ultramafic silicate melts from our study plotted as a function of pressure. The gray and green shaded fields delineate the range of $\mathrm{Ni}$ and Co partition coefficients, respectively from the previous metal-silicate experiments (Thibault and Walter, 1995; Li and Agee, 1996; O'Neill et al., 1998; Kegler et al., 2008). Close correspondence between the $D_{\mathrm{Ni}}^{\text {metal } / \text { silicate }}$ and $D_{\mathrm{Co}}^{\mathrm{meta} / \text { silicate }}$ of our study and those from literature suggests that our new metal-silicate experiments achieved expected magma ocean equilibria. The error bars in partition coefficients are obtained by propagating $1 \sigma$ uncertainties in metal and silicate melt concentrations. (For interpretation of the references to color in this figure legend, the reader is referred to the web version of this article.) 
In order to ensure that our metal-silicate experiments for carbon partitioning reached the desired equilibrium, we compared $D_{\mathrm{Ni}}^{\mathrm{metal} / \text { silicate }}$ and $D_{\mathrm{Co}}^{\mathrm{metal} / \text { silicate }}$ from our experiments with the literature data. Fig. 3 shows that our data present the well-established trends of decreasing compatibility of $\mathrm{Ni}$ and $\mathrm{Co}$ in metallic melts with increasing pressure in the $P-T$ range of our investigation (Thibault and Walter, 1995; Li and Agee, 1996; O’Neill et al., 1998; Kegler et al., 2008), suggesting that the experiments capture the expected equilibrium between metallic and silicate melts in a magma ocean.

\subsection{Water content of silicate melts}

Measured content of hydrogen, expressed as water, in our basaltic glasses with pre-dried starting silicate compositions vary from 0.29 to $0.67 \mathrm{wt} . \%$. Whereas a single glassy sample of komatiitic composition yields $0.54 \mathrm{wt} . \%$ $\mathrm{H}_{2} \mathrm{O}$. Pre-dried starting mixes that quench to heterogeneous dendritic texture (peridotite and komatiite in graphite capsule and samples run in $\mathrm{MgO}$ capsule) also yield similar range $(0.49-0.72 \mathrm{wt} . \%)$ of total water contents. In contrast, the Knippa composition that was not dried prior to the experiments (Knippa-H) produced glasses with $1.76-1.89$ wt. $\% \mathrm{H}_{2} \mathrm{O}$. Our pre-dried alkalic and tholeiitic basaltic glasses contain similar or lower level of water contamination compared to what have been measured in existing experimental studies where nominally water-free conditions were targeted in the presence of another volatile species (e.g., Stanley et al., 2011; Filiberto et al., 2012).

\subsection{Carbon concentration in Fe-rich metallic melts}

Carbon contents of metallic melts provide solubility limits for the experiments in graphite capsules and vary according to the partition coefficient between metal melt

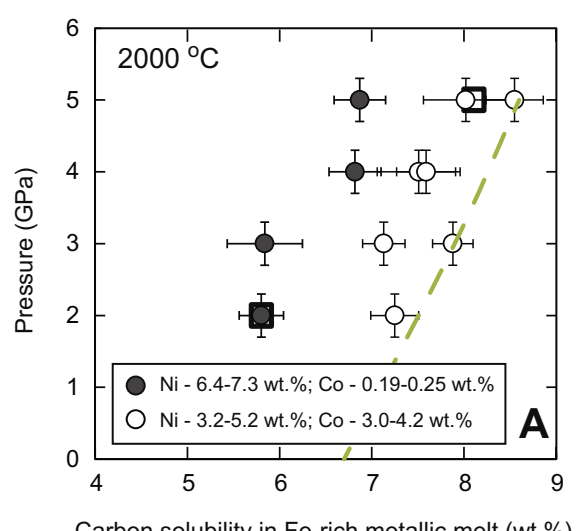

Carbon solubility in Fe-rich metallic melt (wt.\%) and silicate melt for the experiments that were contained in $\mathrm{MgO}$ capsules and were under-saturated with respect to graphite.

Because one set of our experiments in graphite capsules were conducted at a single temperature of $2000{ }^{\circ} \mathrm{C}$ and another set at $1600{ }^{\circ} \mathrm{C}$, the measured carbon concentrations in metallic melts constrain the pressure dependence of C-solubility over the pressure range of $2-5 \mathrm{GPa}$ at $2000^{\circ} \mathrm{C}$ and $1-$ $3 \mathrm{GPa}$ at $1600{ }^{\circ} \mathrm{C}$. Metal melts for all compositions ('core 1', 'core 2', and 'core 3'; Table 1) show modest increase in $\mathrm{C}$ solubility with increasing pressure (Fig. 4). At $2000{ }^{\circ} \mathrm{C}$, alloy melts with higher range of $\mathrm{Ni}$ contents and minor Co contents ('core 2') show C increase from $5.8 \pm 0.2 \mathrm{wt} . \%$ at $2 \mathrm{GPa}$ to $6.9 \pm 0.3 \mathrm{wt} . \%$ at $5 \mathrm{GPa}$ whereas alloy melts with lower $\mathrm{Ni}$ contents and 3.0 4.2 wt. \% Co contents (with 'core 1') show C solubility to increase from $\sim 7.1 \mathrm{wt} . \%$ at $2 \mathrm{GPa}$ to $\sim 8.5 \mathrm{wt} . \%$ at $5 \mathrm{GPa}$. At $1600^{\circ} \mathrm{C}$ as well, alloy melts of a given $\mathrm{Ni}$ content show modestly higher carbon content at $3 \mathrm{GPa}$ compared those at $1 \mathrm{GPa}$. But similar to the dataset at $2000^{\circ} \mathrm{C}$, carbon solubility of alloy melts vary inversely with $\mathrm{Ni}$ contents (Fig. 4). The trends of increasing CCGS for the metallic melts with increasing pressure were observed irrespective of the analytical techniques (EPMA versus SIMS) employed. We also note that, unlike in the study of Dasgupta and Walker (2008) where carbon solubility of Fe-rich metal melt (at $2 \mathrm{GPa}$ ) was obtained from analysis of quenched melt in direct contact with graphite capsules and therefore perhaps had more uncertainty in distinguishing quench versus equilibrium graphite blades, in the present study quenched metal melts were separated from the capsule walls by surrounding silicate melt matrix and hence the analyzed volumes have less likelihood of being affected by the presence of capsule materials.

For experiments in $\mathrm{MgO}$ capsules, the metallic melts have carbon concentrations well below the saturation values and range from 1.6 to $4.2 \mathrm{wt} . \%$.

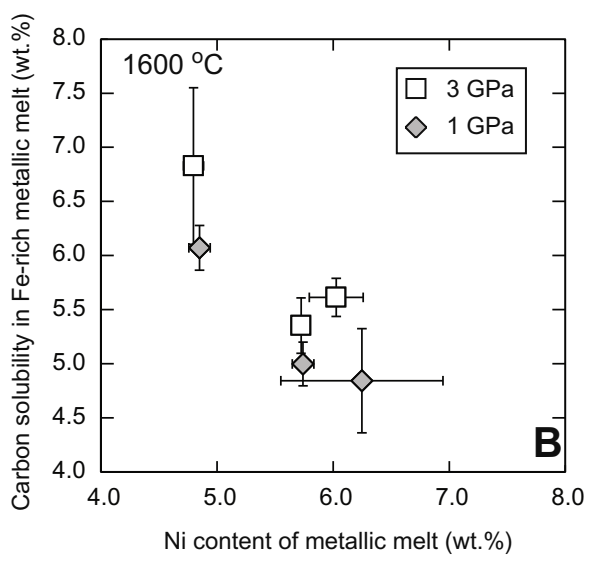

Fig. 4. Carbon concentration at graphite saturation (CCGS) for Fe-rich metallic alloy melts as a function of pressure at a constant temperature of $2000^{\circ} \mathrm{C}$ (A) and as a function of metal melt Ni content at a constant temperature of $1600^{\circ} \mathrm{C}(\mathrm{B})$. The data in (A) are grouped into two classes, one with metal alloy melt having higher Ni contents and minor Co contents (open circles) and another with metal alloy melt having lower Ni contents and similar Co contents (closed circles). Both compositional groups have similar sulfur contents and show a modest increase in CCGS with increasing pressure at a fixed temperature. Also shown for comparison are thermodynamic predictions of C-solubility in metallic iron melt (dashed line) at the same temperature after Siebert et al. (2011) and measured C-solubility in metallic-Fe melt at 2 (Dasgupta and Walker, 2008) and 5 GPa (Nakajima et al., 2009) (open squares). On average, all the measured data suggest somewhat lower solubility of carbon in Fe-rich metallic melt compared to the prediction of Siebert et al. (2011). 


\subsection{Carbon content in silicate melts at graphite and metal melt saturation}

The carbon content of basaltic silicate melt at graphite saturation, CCGS varies as a function of (1) temperature, (2) pressure, (3) silicate melt composition (NBO/T), and (4) water content of silicate melt (Fig. 5).

(1) From 1600 to $2000{ }^{\circ} \mathrm{C}$ at the same pressure of $3 \mathrm{GPa}$ and similar melt composition (MAR and ThB), CCGS increases from $11 \pm 1 \mathrm{ppm} \mathrm{C}$ (Run No. G212) to $24 \pm 1$ ppm C (Run No. BB-929).

(2) CCGS for basaltic melt at a given temperature and composition decreases with increasing pressure (Table 3; Fig. 5) - from $55 \pm 2$ to $19 \pm 2$ ppm C for basaltic composition MAR at $2000{ }^{\circ} \mathrm{C}$ from 2 to $4 \mathrm{GPa}$, from $24 \pm 1$ to $12 \pm 1 \mathrm{ppm} \mathrm{C}$ for basaltic composition $\mathrm{ThB}$ at $1600{ }^{\circ} \mathrm{C}$ from 1 to $3 \mathrm{GPa}$, from $74 \pm 4$ to $39 \pm 2$ ppm C for alkali basalt composition Knippa at $1600^{\circ} \mathrm{C}$ from 1 to $3 \mathrm{GPa}$, and from $108 \pm 2$ to $58 \pm 1 \mathrm{ppm} \mathrm{C}$ for Knippa-H at $1600^{\circ} \mathrm{C}$ from 1 to $3 \mathrm{GPa}$.

(3) Comparison of ThB and Knippa data reveals that at a given pressure, temperature, and extent of melt hydration, CCGS also increases with increasing melt depolymerization, expressed as NBO/T. For example, at $1 \mathrm{GPa}, 1600{ }^{\circ} \mathrm{C} \mathrm{ThB}$ contains $24 \pm 1 \mathrm{ppm} \mathrm{C}$ and Knippa contains $74 \pm 4 \mathrm{ppm}$ C. Similarly, at $3 \mathrm{GPa}, 1600^{\circ} \mathrm{C} \mathrm{ThB}$ contains $12 \pm 1 \mathrm{ppm} \mathrm{C}$ and Knippa contains $39 \pm 1 \mathrm{ppm} \mathrm{C}$. We note that this increase in CCGS with increasing melt $\mathrm{NBO} / \mathrm{T}$ is not linked to melt water content, as these experiments compared here all contain $0.3-0.5 \mathrm{wt} . \% \mathrm{H}_{2} \mathrm{O}$ (Table 3).

(4) CCGS of a basaltic melt at a given $P-T$ also increases with increasing dissolved hydrogen content. The experiments with unfired Knippa, containing $\sim 1.7$ wt. $\% \mathrm{H}_{2} \mathrm{O}$ (total hydrogen expressed as 'water'), at 1 and $3 \mathrm{GPa}, 1600^{\circ} \mathrm{C}$ yield CCGS of $108 \pm 2$ and $58 \pm 1$ ppm $\mathrm{C}$ respectively, whereas the experiments with pre-dried Knippa (Knippa-H), containing 0.30.4 wt. $\% \mathrm{H}_{2} \mathrm{O}$, at the same $P-T$ conditions yield $74 \pm 4$ and $39 \pm 2$ ppm C (Table 3; Fig. 5).

Significant uncertainty remains for the carbon concentrations measured in quenched komatiitic and peridotitic liquid because these compositions did not quench to homogeneous glasses and ion probe analysis relies on average ionization of sample volumes that comprise both quenched dendritic crystals and interstitial glassy patches. Our measured CCGS for peridotitic melt varies from $703 \pm 100$ to $220 \pm 50 \mathrm{ppm}$ from 3 to $5 \mathrm{GPa}$ at $2000^{\circ} \mathrm{C}-$ a similar trend of pressure dependence as observed for the basaltic glasses, although the absolute concentrations are about an order of magnitude higher than those in basaltic. Komatiitic quenched melt MT-7C ( 90-600 ppm C), however, does not yield the trend of decreasing CCGS with increasing pressure, perhaps owing to uncertainty in measured carbon content. In Fig. 5c we plot CCGS versus melt
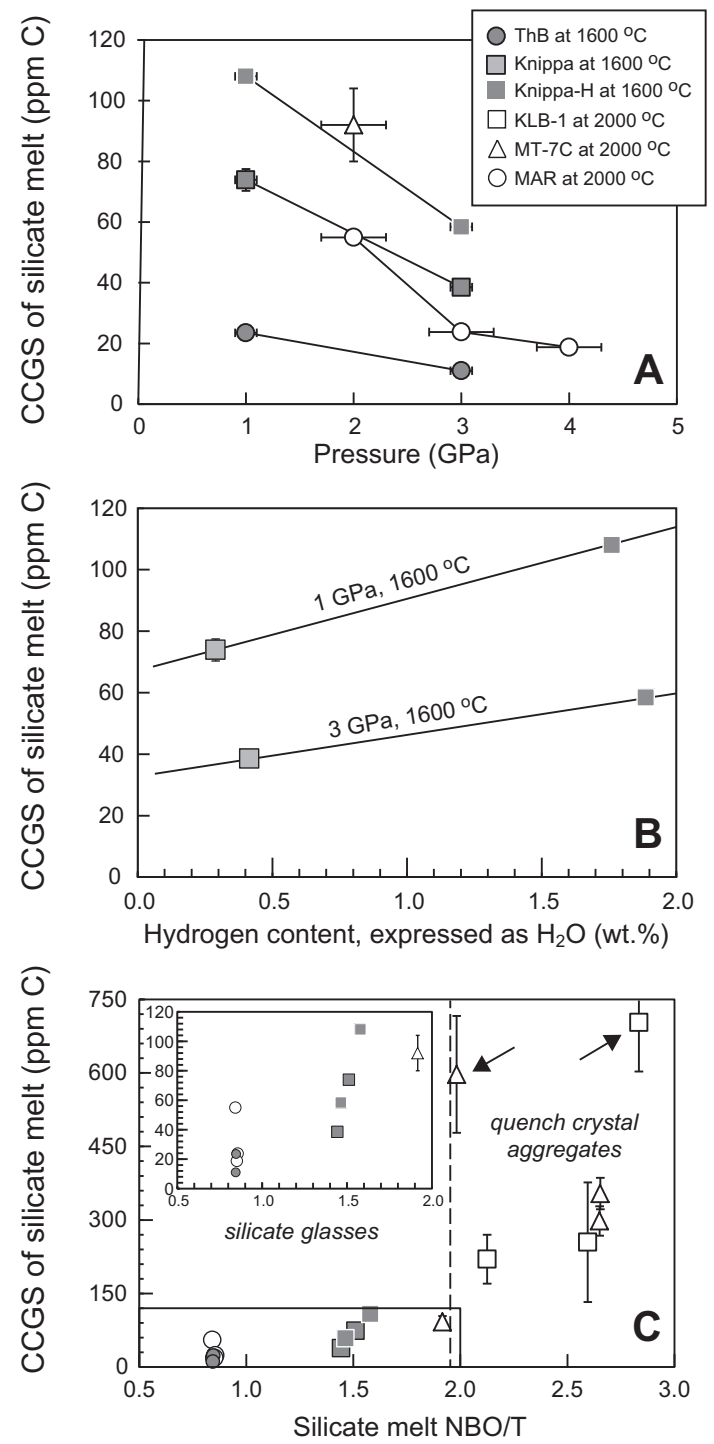

Fig. 5. Carbon concentration at graphite saturation, CCGS for mafic-ultramafic melt from our metal-saturated experiments. (A) The effect of pressure on CCGS of experimental glasses. (B) The effect of melt hydrogen content (expressed as weight percent water) on CCGS of glassy melts of similar composition and at the same temperatures. (C) The effect of melt depolymerization index, NBO/ $\mathrm{T}$ on CCGS of basaltic, komatiitic, and peridotitic melts. The dashed vertical line separates melt compositions that produced glasses (to the left of the line) and those that generated dendritic crystal aggregates (to the right of the line). The trend of increasing CCGS with increasing NBO/T from the glassy samples alone lend credibility that the higher CCGS of more depolymerized melts may not be analytical artifacts. Two experiments, marked with black arrows, however, do show anomalously high CCGS values suggesting that SIMS analyses of these experiments likely have suffered contamination and/ or biased by heterogeneity in the quench assemblage. The inset in (C) shows blown-up portion of the plot with data from silicate glasses only.

polymerization index, NBO/T from all graphite-saturated experiments, distinguishing whether the CCGS was obtained from silicate glasses or from quench dendritic 
mattes. It can be seen that the trend of increasing CCGS with increasing $\mathrm{NBO} / \mathrm{T}$ can be established from the glassy samples alone, thereby ruling out the possibility that the positive dependence of CCGS on melt composition is affected by higher carbon content in quench modified komatiitic and peridotitic melt. However, Fig. 5c also reveals that SIMS analysis of quenched silicate melt pool in at least two of the experiments, BB-901 and BB-919, which yielded $\sim 600-700$ ppm C, may indeed have been affected by carbon contamination or local enrichment owing to quench and thus are not used for further discussion and partition coefficient calculations.

\subsection{Raman spectra of basaltic melts at graphite and metal melt saturation}

Raman spectra of quenched glasses, where available, show asymmetric bands at $\sim 3600$ and $\sim 4100 \mathrm{~cm}^{-1}$ indicating the presence of hydrogen as $\mathrm{OH}^{-1}$ and molecular $\mathrm{H}_{2}$, respectively (Fig. 6). A distinct peak at $\sim 2900 \mathrm{~cm}^{-1}$ indicates the presence of $\mathrm{C}-\mathrm{H}$ stretching bond as part of either molecular $\mathrm{CH}_{4}$, or other hydrocarbon groups such as $-\mathrm{CH}_{3}$ or $-\mathrm{CH}_{2}-$ bonded to silicate network (Mysen et al., 2009; Mysen and Yamashita, 2010). Also, peaks at $\sim 3290 \mathrm{~cm}^{-1}$ are present in all the samples, which likely reflect the presence of $\mathrm{C}-\mathrm{H}$ bonds as part of alkyne groups $(\mathrm{C} \equiv \mathrm{C}-\mathrm{H})$ (Mysen et al., 2009). Comparison of spectra of Knippa versus Knippa-H samples shows that the former not only has lower water peak height at $\sim 3600 \mathrm{~cm}^{-1}$ but also lower peak height at $\sim 2900 \mathrm{~cm}^{-1}$ suggesting less carbon present as methane-like species for samples with lower hydrogen content (Fig. 6). Comparison of run BB930 (MAR at $4 \mathrm{GPa}$ ) and BB932 (MAR at $2 \mathrm{GPa}$ ) also shows that basaltic glasses with similar composition $(\mathrm{NBO} / \mathrm{T})$ and extent of hydration yield higher $2900 \mathrm{~cm}^{-1}$ peak height at lower pressures.

\section{DISCUSSION}

The chief goal of our work is to constrain $D_{\mathrm{C}}^{\text {metal/silicate }}$ at conditions and compositions relevant for a magma ocean. But before we derive $D_{\mathrm{C}}^{\mathrm{metal} / \text { silicate }}$, it is critical to evaluate how carbon is dissolved in silicate melt under the conditions of our experiments because the nature of carbon speciation and solubility in silicate melt depends critically on $P-T-X-$ $f \mathrm{O}_{2}$, thus having a key influence on magma ocean carbon fractionation. It is also important to evaluate whether the SIMS determination of carbon in quench silicate melts is consistent with the known solubility of carbon under similar conditions.

\subsection{Carbon solution in silicate melt at reduced magma ocean conditions and carbon content at graphite saturation, CCGS}

Because most of our experiments were conducted in graphite capsules, two key parameters affecting the equilibrium partition coefficient of carbon between metal alloy melt and silicate melt are the solution mechanism (speciation) and carbon content of reduced mafic-ultramafic melts at graphite saturation.
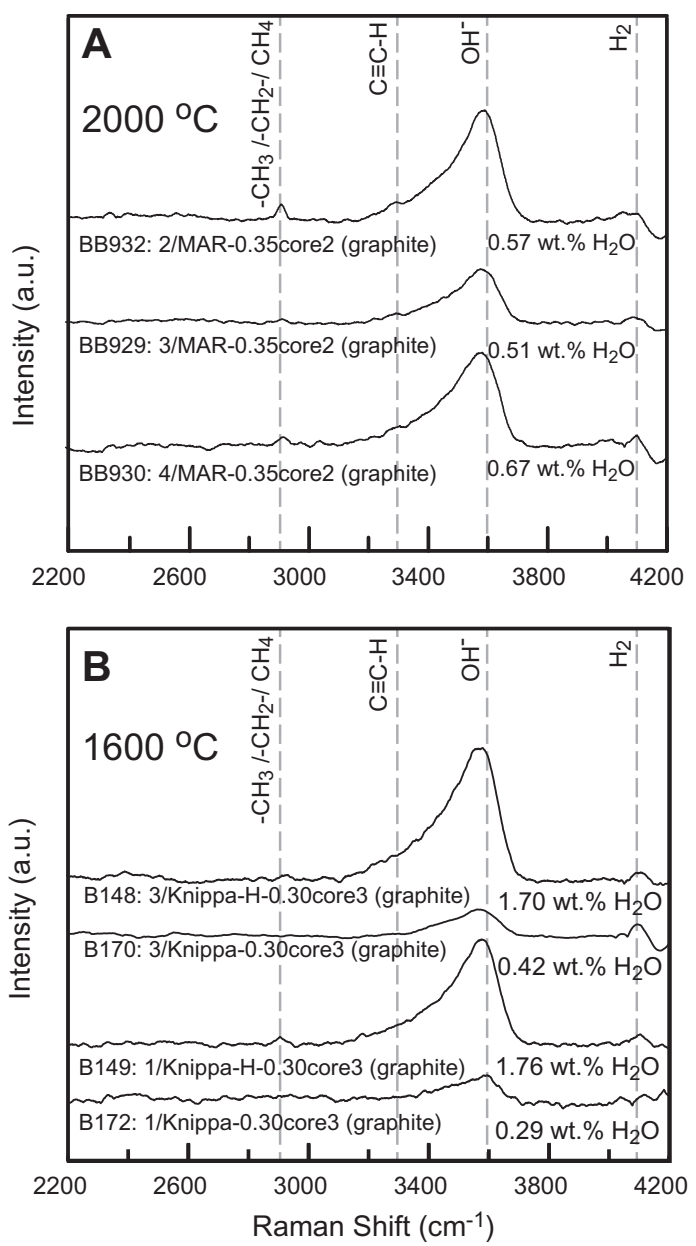

Fig. 6. (A) Baseline-corrected Raman spectra of quenched tholeiitic glasses at graphite saturation and in equilibrium with an immiscible Fe-rich metallic melt. All the experiments plotted are performed with basalt composition MAR (+metal composition 'core 2') at graphite saturation at a pressure of 2-4 GPa at $2000{ }^{\circ} \mathrm{C}$. Asymmetric peaks at frequency of $\sim 3600 \mathrm{~cm}^{-1}$ and at $\sim 4100 \mathrm{~cm}^{-1}$ suggest the presence of hydrogen in silicate melts as both $\mathrm{OH}^{-1}$ and as molecular hydrogen, $\mathrm{H}_{2}$. Small but definite presence of peaks at frequency of $\sim 2900 \mathrm{~cm}^{-1}$ suggests carbon dissolution in melts as $\mathrm{CH}_{4}$ or as methyl group attached to silicate network. Band at $\sim 3290 \mathrm{~cm}^{-1}$ is presumably caused by C-H stretching in an alkyne group (Mysen et al., 2009). The height of the peak at frequency $\sim 2900 \mathrm{~cm}^{-1}$ for the $2 \mathrm{GPa}$ experiment is higher than those at 3 and $4 \mathrm{GPa}$, consistent with higher carbon concentration at the former condition with respect to the latter. (B) Baseline-corrected Raman spectra of quenched alkalic basalt glasses with composition Knippa at graphite and Fe-rich metallic melt saturation. All the experiments plotted are performed at $1600{ }^{\circ} \mathrm{C}$ and at 1 and $3 \mathrm{GPa}$. For each $P-T$ conditions, spectra for both pre-dried Knippa composition and undried Knippa composition are plotted. Undried Knippa compositions at both conditions show greater peak height for $\sim 3600 \mathrm{~cm}^{-1}$ band suggesting higher total water content. Compared to their predried counter parts, the experiments with higher 'water' peak height also show more prominent peak for the $\sim 2900 \mathrm{~cm}^{-1}$ band, suggesting enhanced dissolved methane for melts that are more hydrous. Individual spectra in both (A) and (B) are offset for clarity. 


\subsubsection{Solution mechanism of carbon in reduced mafic- ultramafic melt}

In order to fully understand the possible range of metalsilicate carbon partition coefficient, the speciation of carbon in the silicate melt needs to be constrained. Based on the spectroscopic studies of natural and experimental basaltic glasses, carbon is known to dissolve in mafic-ultramafic melts chiefly as carbonate anions bonded to network modifiers such as $\mathrm{Ca}^{2+}, \mathrm{Mg}^{2+}$, and $\mathrm{Fe}^{2+}$ (e.g., Blank and Brooker, 1994). However, $\mathrm{CO}_{2}$ solubility in basaltic magmas decreases with decreasing oxygen fugacity (Pawley et al., 1992; Thibault and Holloway, 1994; Mysen et al., 2011) and carbonates may not be the dominant carbon species of interest for metal saturated, reduced magma oceans. The possible reduced species of carbon in silicate melt include $\mathrm{CO}, \mathrm{CH}_{4}$ (or other higher order $\mathrm{C}-\mathrm{H}$ molecules or groups bonded to silicate network), and structural units such as Si-C (Kadik et al., 2004; Mysen and Yamashita, 2010; Mysen et al., 2011). This is especially true for melts that have a small concentration of dissolved water (Mysen et al., 2009, 2011; Mysen and Yamashita, 2010) or modest $f \mathrm{H}_{2}$, which are related to oxygen fugacity. Our Raman spectroscopic data validate that hydrogenated species of carbon, such as molecular $\mathrm{CH}_{4}$ or methyl groups bonded to silicate network are present in the basaltic melts and the peak height correlates positively with the total hydrogen content and negatively with pressure (Fig. 6). The Raman spectra of silicate glasses also reveal possible alkyne group $(\mathrm{C} \equiv \mathrm{C}-\mathrm{H})$ as part of dissolved hydrogenated species of carbon (Socrates, 2004) (Fig. 6). No convincing spectral feature of a $\mathrm{Si}-\mathrm{C}$ structural unit or carbonyl group (Wetzel et al., 2012) was observed in our Raman spectra, suggesting that if such species exist in silicate melt, different oxygen and hydrogen fugacity conditions and pressure lower than that explored in our study are required.

Although our Raman spectra do show the presence of dissolved $\mathrm{CH}_{4}$ and alkyne groups in our experimental glasses, carbonate groups are still likely to be an important contributor to the total carbon budget. For example, the experiments on similar compositions but with higher total hydrogen content (Knippa-H) do show higher abundance of dissolved water (Fig. 6b). This suggests that the higher total carbon content of Knippa-H silicate melts compared to those of Knippa silicate melts also likely is contributed by higher dissolved carbonates, because dissolved water depolymerizes melt and enhances carbonate solubility (e.g., Eggler and Rosenhauer, 1978; Behrens et al., 2009) according to the following reactions:

$$
\begin{aligned}
& \underset{(\text { melt })}{\mathrm{O}^{0}}+\underset{\text { (vapor) }}{\mathrm{H}_{2} \mathrm{O}}=\underset{\text { (melt) }}{2\left(\mathrm{O}^{-} \cdot \mathrm{H}^{+}\right)} \\
& \underset{\text { (vapor) }}{\mathrm{CO}_{2}}+\underset{\text { (melt) }}{2 \mathrm{O}^{-}}=\underset{\text { (melt) }}{\mathrm{O}^{0}}+\underset{\text { (melt) }}{\mathrm{CO}_{3}^{2-}}
\end{aligned}
$$

where $\mathrm{O}^{0}$ and $\mathrm{O}^{-}$are bridging and singly bound non-bridging oxygen, respectively (Eggler and Rosenhauer, 1978). Thus increasing hydration of graphite/diamond-saturated magma ocean may cause increased carbon solution not only owing to higher $f \mathrm{H}_{2}$, through the following reaction:

$\underset{\text { (graphite/diamond) }}{\mathrm{C}}+\underset{\text { (vapor) }}{2 \mathrm{H}_{2}}=\underset{\text { (melt) }}{\mathrm{CH}_{4}}$ but also owing to higher $f \mathrm{H}_{2} \mathrm{O}$, which should cause generation of more non-bridging oxygen in the melt (Eq. (4)), thus facilitating greater carbonate solubility (Eq. (5)).

Because it is expected that CCGS of silicate melt, especially at reduced, core-forming conditions is likely controlled by the sum of equilibrium concentration of individual species, i.e., $\mathrm{CO}_{2}$ (as dissolved carbonates) and $\mathrm{C}-\mathrm{H}$ groups such as $\mathrm{CH}_{4}$, in the following, we constrain the relative contribution of different carbon species in our experiments. Parameterization by Holloway et al. (1992) allows calculation of $\mathrm{CO}_{2}$ (as carbonate) content in basaltic melt at graphite saturation and over the range of pressure, temperature, and $\mathrm{fO}_{2}$ conditions relevant for a shallow magma ocean. We plot in Fig. 7, the calculated carbon content of basaltic melt at graphite saturation present as $\mathrm{CO}_{2}$ (Holloway et al., 1992) at the $P-T-f \mathrm{O}_{2}$ conditions relevant for our experiments against the total measured carbon in our glassy samples measured using SIMS. It can be seen that our measured values of total carbon content in silicate glasses are consistently higher. If the calibration of Holloway et al. (1992), which is for tholeiitic basalts, applies to all of our melt compositions, the MAR glasses at $2000{ }^{\circ} \mathrm{C}$ and $\Delta \mathrm{IW}$ of -1.6 to -1.7 have $20-50 \%$ of its carbon present as reduced species, whereas the ThB and Knippa glasses at $1600{ }^{\circ} \mathrm{C}$ and $\Delta \mathrm{IW}$ of -1.5 have $85-87 \%$ and $95-98 \%$ of

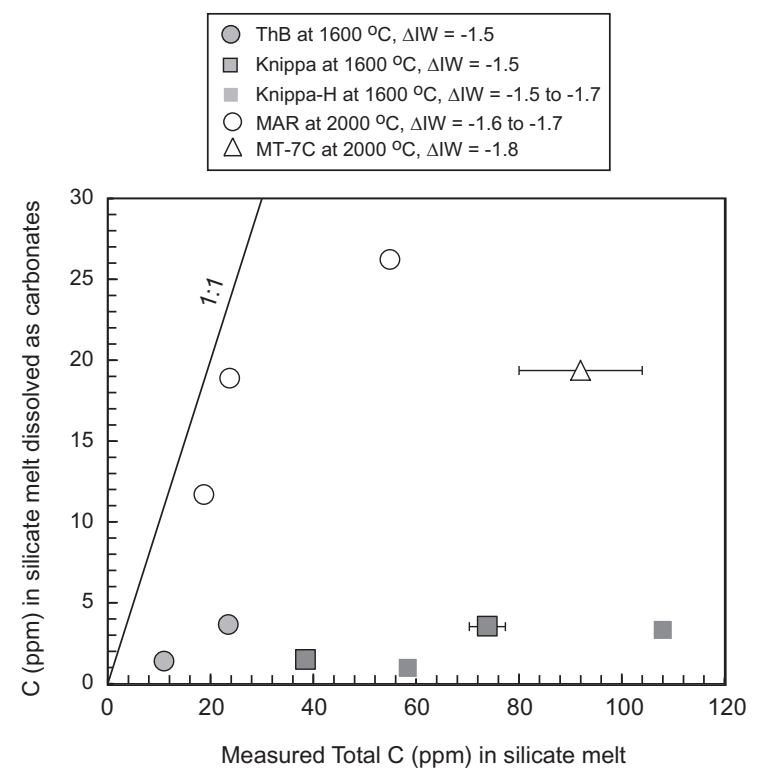

Fig. 7. Calculated concentration of carbon dissolved as carbonates in basaltic and komatiitic glasses at graphite saturation and at the experimental $P-T-f \mathrm{O}_{2}$ conditions, based on the model of Holloway et al. (1992) plotted as a function of total dissolved carbon measured using SIMS. All the experiments plot below the 1:1 line, suggesting that our silicate glasses also contain carbon in the form of non-carbonated species. In order to have a robust comparison of calculated carbon (dissolved as $\mathrm{CO}_{2}$ ) versus measured carbon, only the experiments with glassy melt pools are plotted. The difference between total estimated carbon and carbon as carbonates is even higher for peridotitic and other komatiitic samples that did not quench to a glass. The error bar in measured carbon represents 1 sigma uncertainty based on replicate SIMS analyses. Where absent, the error bar is smaller than the size of the symbols. 
their carbon present as reduced species. Similarly, the single komatiitic glass at $2000{ }^{\circ} \mathrm{C}$ and $\Delta \mathrm{IW}$ of -1.8 appears to have $\sim 80 \%$ of its CCGS contributed by reduced species. If CCGS values for peridotitic melt, obtained from quenched dendritic crystal aggregates, are considered (not plotted), the difference between the model prediction of $\mathrm{CO}_{2}$ content and the measured total carbon is even more dramatic. These differences may owe in part to the fact that the model of Holloway et al. (1992) was developed for a tholeiitic basalt and hence does not apply to more depolymerized (and hydrous) basaltic melt compositions. Indeed the CCGS values obtained for MAR or ThB compositions are consistently lower compared to the ones for Knippa and MT-7C or KLB-1, suggesting that the carbon solubility at graphite saturation is higher for more depolymerized melts (Fig. 7). However, the mismatch of ThB and MAR basalts CCGS with the modeled $\mathrm{CO}_{2}$ solubility clearly indicate that non-oxygenated species such as methane or alkyne group are important carbon species in our experimental melts (Fig. 7). Alternatively, the model of Holloway et al. (1992) may underestimate the $\mathrm{CO}_{2}$ solubility in reduced magmas at conditions similar to our experiments, especially given the fact that the $\mathrm{fO}_{2}$ dependence of $\mathrm{CO}_{2}$ solubility in the thermodynamic model of Holloway et al. (1992) is only verified with experiments at distinctly higher range of $\mathrm{fO}_{2}$ (Pawley et al., 1992; Thibault and Holloway, 1994). We note that although our measured carbon contents in glasses exceed the abundance of carbon expected to be dissolved as carbonates (Fig. 7), the trends of decreasing $\mathrm{C}$ concentration with increasing pressure and increasing $\mathrm{C}$ concentration with increasing temperature at a constant $\mathrm{fO}_{2}$ are exactly what is predicted for the solubility of $\mathrm{CO}_{2}$ at graphite saturation (Holloway et al., 1992). Hence, even though the total carbon solubility in our experimental melts appear more than those predicted by Holloway et al. (1992) model, dissolved carbonates may still significantly control the systematics of carbon solubility trends at reduced, core-forming magma ocean conditions. However, it is also possible that the other reduced species such as $\mathrm{CH}_{4}$ also show similar dependence to $P-T-f \mathrm{O}_{2}$ in the presence of graphite. In the absence of graphite-present, methane solubility data for natural basaltic melt, these possibilities are difficult to evaluate. Future experimental studies will have to measure the concentration of $\mathrm{CO}_{2}$, dissolved as carbonates, in strongly reduced basaltic glasses of variable compositions to constrain the relative importance of carbonates versus other reduced species in the total inventory of carbon in a magma ocean.

How species-specific carbon solubility changes as a function of melt composition or structure at low $f \mathrm{O}_{2}$ is poorly known. However, the increase of CCGS from tholeiitic basalt $\mathrm{ThB}$ to Knippa under similar $P-T$ condition and hydrogen content (Fig. 5a and c) suggests that the solubility of one or more carbon species in the melt is enhanced with increased non-bridging oxygen over tetrahedral cations in the melt. Increased solubility of carbonates with higher degree of melt depolymerization at oxidized conditions is well established (Brooker et al., 2001), our data may indicate that such trend holds true even for graphite saturated conditions at $f \mathrm{O}_{2}$ as low as IW-1.5 to IW-2.0. It is equally likely, however, that the increased CCGS from tholeiitic to alkalic basalt is entirely contributed by hydrogenated species such as $\mathrm{CH}_{4}$, consistent with the recent observation of Mysen et al. (2009) that NBO/T correlates positively with methane solubility.

\subsubsection{Carbon content of reduced, mafic-ultramafic melts at graphite saturation, CCGS}

Our experiments in graphite capsules constrain the CCGS of silicate melts at oxygen fugacity $\sim \mathrm{IW}-1.5$ to IW-1.8 and between 1 and $5 \mathrm{GPa}$ at $1600-2000{ }^{\circ} \mathrm{C}$. The purpose of this section is to compare our CCGS data with the known values of silicate melt $\mathrm{C}$-solubility at reduced
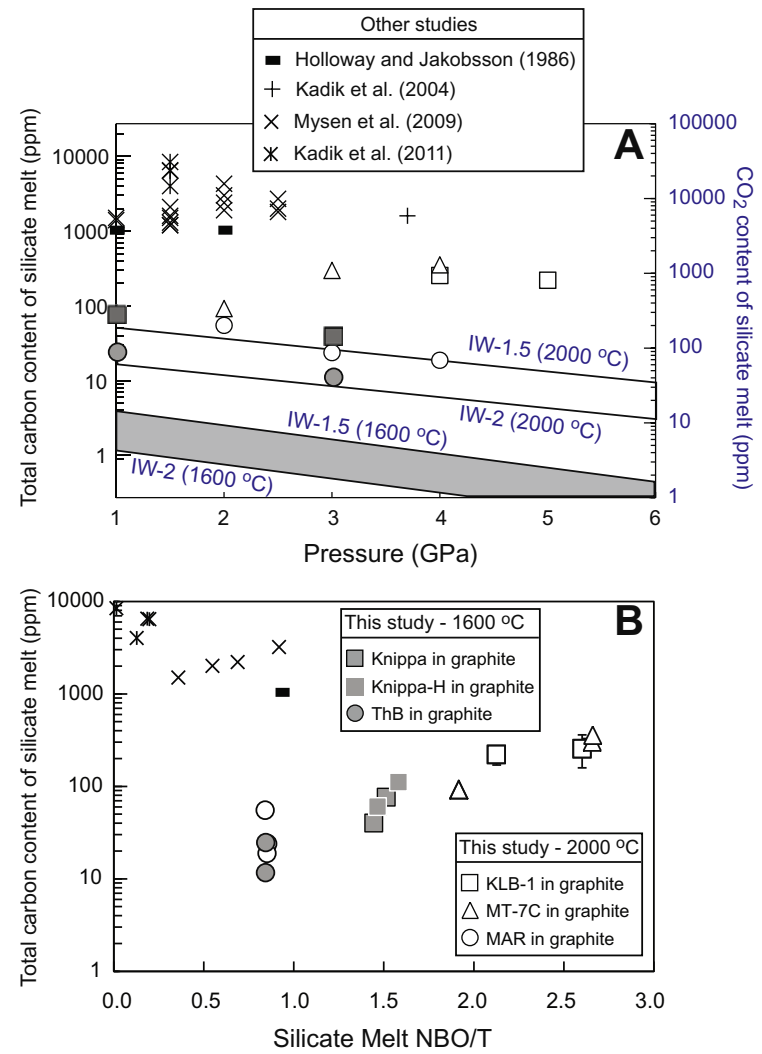

Fig. 8. Carbon concentration of silicate melts at graphite saturation from our metal melt saturated experiments $\left(f \mathrm{O}_{2} \sim \mathrm{IW}-1.5\right.$ to IW-2.0) using tholeiitic basalts (ThB and MAR), alkalic basalt (Knippa and Knippa-H), komatiite (MT-7C), and fertile peridotite (KLB-1) at 1600 and $2000^{\circ} \mathrm{C}$ as a function of pressure (A) and silicate melt depolymerization index, $\mathrm{NBO} / \mathrm{T}(\mathrm{B})$. Also shown for comparison in (A) are estimated content of $\mathrm{CO}_{2}$ at graphite saturation at 1600 and $2000{ }^{\circ} \mathrm{C}$ and between $f \mathrm{O}_{2}$ of IW-1.5 and IW2.0. Both the panels also plot CCGS and methane solubility values obtained in other studies at reduced conditions, which include graphite and methane-rich fluid saturated tholeitic silicate melt at $f \mathrm{O}_{2} \sim \mathrm{IW}$ and $1-2 \mathrm{GPa} / 1200{ }^{\circ} \mathrm{C}$ (Holloway and Jakobsson, 1986; Jakobsson and Holloway, 1986), graphite saturated tholeiitic melt at $f \mathrm{O}_{2} \sim \mathrm{IW}-2.3$ and $1520-1600{ }^{\circ} \mathrm{C}$ (Kadik et al., 2004), graphite saturated Na-Al-Si-Fe melt at $f \mathrm{O}_{2} \sim \mathrm{IW}-1.9$ to $\mathrm{IW}-3.7$ and $1.5 \mathrm{GPa} / 1400{ }^{\circ} \mathrm{C}$ (Kadik et al., 2011), and $\mathrm{CH}_{4}-\mathrm{H}_{2}$ fluid saturated Na-silicate glass at $f \mathrm{O}_{2} \sim \mathrm{IW}$ and $1-2.5 \mathrm{GPa} / 1400{ }^{\circ} \mathrm{C}$ (Mysen et al., 2009). CCGS data for Knippa-H compositions are not plotted in panel (A) for clarity. 
conditions to ensure that our measurements are not affected by quench modification and/or contamination.

Published experimental data on carbon solubility in silicate melts under conditions comparable those of the present experiments are scarce but recent experimental studies did attempt to constrain the solubility of reduced, hydrogenated species of carbon in silicate melts at graphite saturation (Kadik et al., 2004, 2011; Mysen et al., 2009, 2011). Although none of the studies were performed for silicate melt compositions and temperatures relevant for natural magma ocean conditions, it is still useful to compare the CCGS obtained in these studies with the experimental determination presented here. Fig. 8 shows that our measured (total) carbon concentrations in mafic-ultramafic melts are intermediate between the carbon concentrations in reduced silicate melts in equilibrium with $\mathrm{CH}_{4}-\mathrm{H}_{2}$ fluid (Mysen et al., 2009) or graphite and carbon dissolved solely as carbonates (Holloway et al., 1992). It is also observed that although the absolute carbon solubility at methane saturation is significantly higher for $\mathrm{Na}$-silicate melt in the study of Mysen et al. (2009), these authors report similar dependence of carbon solubility on $\mathrm{NBO} / \mathrm{T}$ as found in our study.

\subsection{Partitioning of carbon between metallic and silicate melt - Control of different variables}

Partition coefficients of carbon between Fe-rich metallic melt and silicate melt, $D_{\mathrm{C}}^{\mathrm{meta} / \text { silicate }}$ from our experiments are plotted in Fig. 9. $D_{\mathrm{C}}^{\text {metal } / \text { silicate }}$ from our experiments varies from $4757 \pm 355$ (Run No. G212: $3 \mathrm{GPa}, 1600{ }^{\circ} \mathrm{C}$ with $\mathrm{ThB} /$ core 3 in graphite capsule) to $134 \pm 28$ (Run No. BB-901: $5 \mathrm{GPa}, 2000{ }^{\circ} \mathrm{C}$ with $\mathrm{MT}-7 \mathrm{C} /$ core 1 in graphite capsule) (Table 3 ). $D_{\mathrm{C}}^{\mathrm{metal} / \text { silicate }}$ increases as a function of pressure for experiments with the composition MAR, ThB, Knippa, and Knippa-H in graphite capsules. Similar increase is also noticed for experiments with composition KLB-1 in graphite capsules but experiments with MT-7C produced no such relationship, perhaps owing to the uncertainty in the estimate of silicate melt carbon content (Fig. 9A). The positive effect of pressure on $D_{\mathrm{C}}^{\mathrm{metal} / \text { silicate }}$ likely results from a combined effect of positive pressure dependence of metal melt CCGS and negative pressure dependence of silicate melt CCGS. The former possibly indicates more ideal mixing between $\mathrm{C}$-rich and $\mathrm{C}$-poor liquid along the graphite-saturated liquidus of Fe-rich metallic melt at higher pressures.

Most of our experiments were conducted at two different temperatures of 1600 and $2000{ }^{\circ} \mathrm{C}$, with only one experiment being performed at $2100{ }^{\circ} \mathrm{C}$ (Table 2). But if the comparison between the graphite saturated experiments at $1600{ }^{\circ} \mathrm{C}$ with 'ThB/core 3' and $2000{ }^{\circ} \mathrm{C}$ with 'MAR/core $2^{\prime}$ were to hold, temperature likely has a negative effect on $D_{\mathrm{C}}^{\text {metal/silicate }}$ (Fig. 9A). This effect of temperature appears to hold even for graphite-absent experiments; for example, from 2100 to $2000{ }^{\circ} \mathrm{C} D_{\mathrm{C}}^{\mathrm{metal} / \text { silicate }}$ drops from $\sim 490$ to $\sim 180$ for composition 'MT-7C/core 1'.

$D_{\mathrm{C}}^{\mathrm{metal} / \text { silicate }}$ also appears to correlate negatively with silicate melt NBO/T, i.e., $D_{\mathrm{C}}^{\mathrm{metal} / \text { silicate }}$ derived for compositions MT-7C and KLB-1 are lower compared to those for basal-
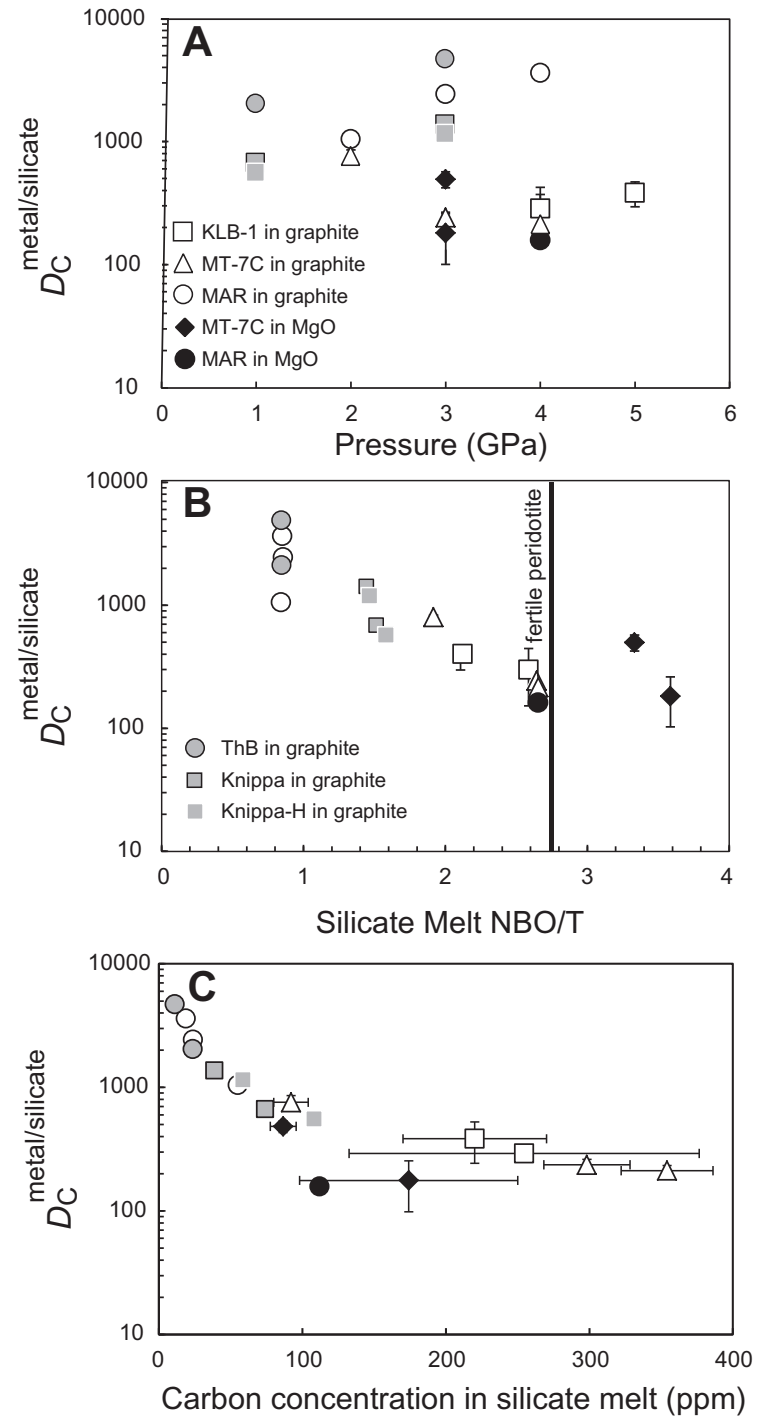

Fig. 9. Experimentally determined partition coefficient of carbon between metallic-Fe-rich melt and mafic-ultramafic silicate melt, $D_{\mathrm{C}}^{\text {metal/silicate }}$ (= mass fraction of carbon dissolved in metallic melt/ mass fraction of carbon dissolved in silicate melt) plotted as a function of pressure (A), depolymerization of silicate melt expressed as $\mathrm{NBO} / \mathrm{T}(\mathrm{B})$, and carbon content of silicate melt (C). Shown for reference in panel (B), is the NBO/T relevant for a fertile peridotite composition. The legends relevant for all the panels are given in (A) and (B).

tic melts (Fig. 9B). Among basaltic glass-bearing runs alone, alkalic basalt Knippa-bearing experiments yield lower $D_{\mathrm{C}}^{\text {metal/silicate }}$ values compared to those containing ThB or MAR, thus ruling out the possibility that lower $D_{\mathrm{C}}^{\mathrm{metal} / \text { silicate }}$ values for depolymerized silicate melts might be analytical artifact of SIMS analysis in heterogeneously quenched melt pool.

Comparison of experiments with Knippa and Knippa-H suggests that $D_{\mathrm{C}}^{\mathrm{metal} / \text { silicate }}$ also diminishes slightly (from 676 to 573 at $1 \mathrm{GPa} / 1600{ }^{\circ} \mathrm{C}$ and from 1390 to $\sim 1170$ at $3 \mathrm{GPa} / 1600{ }^{\circ} \mathrm{C}$; Table 3 ) as the silicate melt hydration increases from $2890-4150 \mathrm{ppm}$ to $17610-18870 \mathrm{ppm} \mathrm{H}_{2} \mathrm{O}$. 
Given the fact that $\mathrm{H}_{2} \mathrm{O}$ solubility in mafic-ultramafic melt is higher than that of $\mathrm{CO}_{2}$ (Dixon et al., 1995) we argue that terrestrial magma ocean must have contained sufficient water to stabilize hydrocarbon species in the same. Therefore although our experiments with lower water content had $>1000$ ppm $\mathrm{H}_{2} \mathrm{O}$, it is likely that terrestrial magma ocean with as low as 100 ppm $\mathrm{H}_{2} \mathrm{O}$ (Elkins-Tanton, 2012) would still have hydrocarbon species at metal saturation and thus would have had CCGS not too different from our estimation (Fig. 5B). However, if the Earth's magma ocean was completely dry then the effective $D_{\mathrm{C}}^{\text {metal/silicate }}$ would likely be even higher.

No particular correlation of $D_{\mathrm{C}}^{\mathrm{metal} / \text { silicate }}$ is noticed with metallic melt carbon content (not shown), suggesting that carbon saturation in metallic melt has no direct influence on $D_{\mathrm{C}}^{\mathrm{metal} / \text { silicate }}$. Similarly, within the range of our metal melt compositions, molar $\mathrm{S} / \mathrm{C}$ ratio, which varies from 0 to $\sim 0.44$ in our study, does not show any obvious control on $D_{\mathrm{C}}^{\mathrm{metal} / \text { silicate }}$ (not shown). But $D_{\mathrm{C}}^{\mathrm{metal} / \text { silicate }}$ appears to correlate negatively with silicate melt carbon content (Fig. 9C). More experiments, especially at variable temperature, oxygen fugacity, and over a wider pressure range are needed to fully parameterize the effect of various variables on $D_{\mathrm{C}}^{\text {metal/silicate }}$.

\subsection{The origin of mantle carbon}

Our measured $D_{\mathrm{C}}^{\mathrm{metal} / \text { silicate }}$ allows us to calculate the expected concentration of carbon in the Earth's metallic core and the mantle residual to core formation. In Fig. 10, we plotted the calculated concentration of carbon in the metallic core and post core formation magma ocean - which crystallizes to form the silicate mantle - as a function of bulk Earth carbon that was available during core-mantle equilibration. The mass balance calculation assumes $32.3 \%$ core mass equilibrating with the $67.7 \%$ molten mantle silicate, the relative masses of the present core and mantle. To cover the range of possible $P-T-\mathrm{X}-f \mathrm{O}_{2}$ conditions relevant for core-mantle equilibration in the terrestrial magma ocean, we show the calculations for $D_{\mathrm{C}}^{\text {metal } / \text { silicate }}=2500,1000$, and 500 . We use $D_{\mathrm{C}}^{\text {metal } / \text { silicate }}$ of 500 as the lower limit because that is close to our lowest $D_{\mathrm{C}}^{\mathrm{metal} / \text { silicate }}$ value and deeper metal-silicate equilibration will further elevate the relevant partition coefficient. Fig. 10 suggests that the average carbon content of the Earth's modern depleted mantle can be matched by equilibrium core-mantle fractionation of carbon if the bulk Earth carbon during core formation was as high as $\sim 0.4-2 \mathrm{wt} . \%$ (4000-20,000 ppm C). In order to generate enriched mantle domains similar to those of some of the ocean island basalt (OIB) source regions, bulk Earth carbon in excess of $2.1 \mathrm{wt} . \%$ and approaching the carbonaceous chondrite value of $3.5 \mathrm{wt} . \%$ is necessary. The abundance of bulk Earth carbon during core-mantle segregation is uncertain, however. In fact, owing to volatility of carbon during the accretion process, the bulk Earth carbon is thought to be significantly less than that of carbonaceous chondrite, and the recent estimate prefers a value $\leqslant 1000 \mathrm{ppm}$. If the avail-

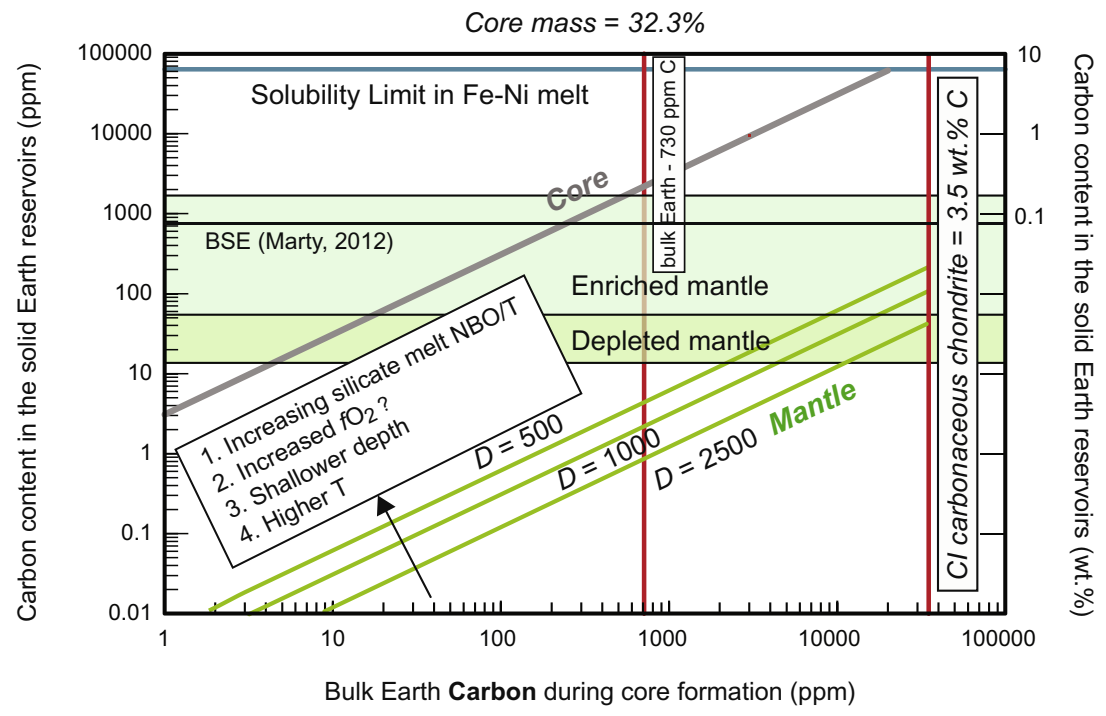

Fig. 10. Carbon content of Earth's core and mantle that result from equilibrium fractionation of the element between metallic and silicate melt in magma ocean conditions. Carbon concentration of the deep Earth reservoirs (core: grey curve and mantle: green curves) are plotted as a function of bulk Earth carbon that participated in the core-mantle fractionation event. The mantle and core concentrations are calculated for three different $D_{\mathrm{C}}^{\text {metal/silicate }}$ values of 2500,1000 , and 500 which likely cover all the possible scenarios (see text for details). Plotted for comparison are the estimated range of modern Earth mantle carbon contents (depleted mantle similar to the source regions of mid-ocean ridge basalts: $\sim 50-200 \mathrm{ppm} \mathrm{CO}_{2}$, enriched mantle similar to the source regions of intraplate ocean island basalt or off-axis seamounts: up to $4000 \mathrm{ppm} \mathrm{CO}$ ) primarily based on volcanic $\mathrm{CO}_{2}$ flux (Dasgupta and Hirschmann, 2010) and the recent estimate of BSE carbon content of $\sim 765$ ppm (Marty, 2012). Also plotted for reference are the carbon abundance in CI chondrite of 3.5 wt.\% (Anders and Grevesse, 1989; Palme and Jones, 2003), estimated bulk Earth carbon content of 730 ppm (McDonough, 2003), and carbon solubility limit for Fe-rich alloy melt at high temperature and low pressure (Dasgupta and Walker, 2008; Nakajima et al., 2009; Siebert et al., 2011). (For interpretation of the references to color in this figure legend, the reader is referred to the web version of this article.) 
able bulk Earth carbon was indeed only $\sim 730 \mathrm{ppm}$ (McDonough, 2003), for the estimated range of $D_{\mathrm{C}}^{\mathrm{metal} / \text { silicate }}$, the mantle residual to core formation could only possess $1-4.5 \mathrm{ppm} \mathrm{C}\left(3.6-16.5 \mathrm{ppm} \mathrm{CO}_{2}\right)$. This is only $7-33 \%$ of the present-day mantle budget of carbon with $50 \mathrm{ppm}$ bulk $\mathrm{CO}_{2}$. If similar to the carbon for enriched mantle domain is used, the contribution of carbon that was left behind after core formation will be even smaller. If the bulk silicate Earth carbon content of $\sim 765 \mathrm{ppm}$ (Marty, 2012) is used then $>99 \%$ of the silicate Earth C needs to be derived from post core-segregation processes. This calculation with our measured $D_{\mathrm{C}}^{\text {metal/silicate }}$ thus suggests that a maximum of $\sim 30 \%$ of the present-day mantle carbon can be primordial, which dates back to the time period prior to the last giant impact. How did the Earth's mantle acquire its present-day carbon budget then, if the bulk Earth carbon is much less than that of carbonaceous chondrite?

\subsubsection{The problem of 'excess' carbon in the mantle- Inefficient core formation or late veneer?}

The problem of 'excess' carbon in the mantle over what would be predicted from equilibrium partitioning between metallic and silicate melt in a magma ocean is reminiscent of the decades-old excess siderophile element problem in core formation. Two plausible solutions that have been explored to explain the siderophile element abundance in the mantle thus can be applied to explain the geochemistry of carbon as well - (1) addition of volatile-rich chondritic 'late veneer' (e.g., Chou, 1978; Wänke, 1981) and (2) inefficient core formation (Jones and Drake, 1986; Newsom and Sims, 1991).

For the first model to be applicable, a carbon-rich late veneer needs to be added. It has been suggested that Earth's volatiles, or at least those of the bulk silicate Earth, were delivered as part of a late veneer that post-dated the last giant impact and core formation (Owen and Bar-Nun, 1995; Dauphas and Marty, 2002). This idea is motivated in part from the premise that Earth may have lost most of its volatiles to space following a giant impact (Abe, 1997; Genda and Abe, 2003) and that the budget of terrestrial volatiles would therefore have to be reintroduced by later additions. Our measured $D_{\mathrm{C}}^{\mathrm{meta} / \text { silicate }}$ suggests that replenishment of mantle carbon may also be required because formation of a metallic core efficiently sequestered the bulk Earth's carbon. But the proposition of carbon addition to the Earth by a 'late veneer' is not devoid of demerits. For example, the late veneer is commonly considered to be chondritic, but if the bulk silicate Earth (BSE) volatiles derive chiefly from a chondritic late veneer, it is difficult to explain why the present-day $\mathrm{H} / \mathrm{C}$ ratio of BSE is superchondritic (Hirschmann and Dasgupta, 2009). Furthermore, CI chondritic materials have carbon isotope compositions of -7 to $-15 \%$ (Kerridge, 1985), distinct from that of the average mantle carbon isotope composition of $-5 \%$ (e.g., Deines, 2002). Thus from the isotopic perspective as well, terrestrial carbon cannot possibly be derived from chondritic late veneer.

The second model calls for incomplete segregation of metallic liquid from the mantle during core formation. It is indeed likely that not the entire batch of metallic liquid efficiently segregated to form the core. Some small fractions of melt may get trapped in the solid mantle matrix, owing to the high dihedral angle of metallic melts in a silicate matrix. Interfacial energies between carbon-bearing metallic alloy melts and mantle minerals are not constrained at present but the knowledge of dihedral angle between many other metallic melt compositions and solid silicates support such a premise (e.g., Minarik et al., 1996; Shannon and Agee, 1996; Terasaki et al., 2007; Mann et al., 2008). Trapped Fe-rich, carbon-bearing metallic melt will eventually stall in the solid mantle owing to high percolation threshold (Watson and Roberts, 2011) and crystallize, providing a source for both siderophile elements and carbon in the mantle. If this was the case, the Earth's lower mantle would start off being more carbon-rich and solid state convection after magma ocean solidification would bring up the lower mantle material with accessory Fe-rich alloy or Fe-rich carbide. For carbon to be released from these reduced accessory phases some form of oxidative process is necessary. The contribution of this form of carbon trapped in alloy or carbide to the total budget of the upper mantle carbon depends critically on its composition. If the trapped metallic melt undergoes batch crystallization and has only modest carbon content, as will be the case if it is derived from equilibrium partitioning in a low bulk carbon environment, then its input to the present-day, upper mantle carbon inventory will be small. For example, if trapped metal with $\sim 0.2 \mathrm{wt} . \% \mathrm{C}$ is only $0.1-1 \%$ of the lower mantle, then such a mantle domain will have only $2-20$ ppm $\mathrm{C}(\sim 7-$ $73 \mathrm{ppm} \mathrm{CO}_{2}$ ). It is not implausible, however, that in some mantle domains, the trapped metallic liquid precipitates an Fe-rich carbide. This can be facilitated by the $\mathrm{Fe}-\mathrm{C}$ eutectic composition becoming increasingly carbon-poor with pressure (Wood, 1993; Lord et al., 2009). If separation of carbide from the remaining metallic liquid takes place, perhaps aided by the presence of silicate partial melts, then some lower mantle domains can be quite carbon-rich. If the lower mantle domain contains $0.1-1 \mathrm{wt} \%$ of Fe7C3, then such parcels can deliver $\sim 84-840 \mathrm{ppm} \mathrm{C}(\sim 310$ $3100 \mathrm{ppm} \mathrm{CO}_{2}$ ) and convective mixing of only $10 \%$ of such mantle mass can contribute $>30-300 \mathrm{ppm} \mathrm{CO}_{2}$ to the Earth's depleted upper mantle.

\subsubsection{Carbon ingassing through magma ocean-atmosphere interaction - Magma ocean 'carbon pump'}

An alternate mechanism for the Earth's mantle gaining carbon post core formation is through interaction of the magma ocean with the Hadean atmosphere. The chemical evolution of the Earth's primitive atmosphere is long debated (Kasting, 1993; Zahnle et al., 2007, 2010) but several authors suggest a persistence of a $\mathrm{CO}_{2}$-rich atmosphere either through degasing of an earlier magma ocean (Elkins-Tanton, 2008) or through formation of a liquid water ocean, which allowed formation of an atmosphere consisting chiefly of $\mathrm{CO}_{2}$ (Zahnle et al., 2007). This can happen when the magma ocean redox state is somewhat more oxidized than what is captured by our studies, i.e., when the dissolved $\mathrm{C}$-species in the melt is dominantly $\mathrm{CO}_{2}$ rather than methane or other reduced species. A recent study sug- 
gests that such an environment was created as early as only within a few hundred million years after core formation (Trail et al., 2011). The depth dependence of $\mathrm{CO}_{2}$ solubility in mafic magma at graphite saturation and near iron-wüstite buffer (Fig. 5) (Holloway et al., 1992) suggests that the storage capacity of a magma ocean at a constant $f \mathrm{O}_{2}$, at shallow depths is greater and diminishes at the expense of graphite/diamond or C-bearing metallic melt with increasing depths. Our CCGS data support this prediction. In this scenario, a magma ocean could dissolve carbon through interaction with an early atmosphere and precipitate graphite/diamond/carbide in its deeper parts as convection brought a batch of magma down to greater depths. Precipitation and sequestration of diamond/carbide/C-bearing metallic melt at depth would lead to under saturation of magma with respect to $\mathrm{CO}_{2}$ and thus upon upwelling would be ready to dissolve more $\mathrm{CO}_{2}$ from the atmosphere. This cycling might have served as an efficient mechanism of magma ocean ingassing, bringing the mantle inventory of carbon up to the present-day value well before magma ocean crystallization. The time scale over which this carbon ingassing mechanism remained operative is unclear at present. The depth dependence of methane solubility is ambiguous (Mysen et al., 2009); thus whether magma ocean could dissolve carbon from a methane rich atmosphere, which was likely the case at least as a long-term transient state right after giant impacts (Zahnle et al., 2010), is unconstrained at present. If $\mathrm{CH}_{4}$ solubility at graphite saturation does not have the same behavior as that of $\mathrm{CO}_{2}$, magma ocean carbon ingassing may have started to operate only after some extent of oxygenation. On the other hand, magma ocean oxidation to the order of quartz-fayalite-magnetite (QFM) buffer should force the magma ocean carbon ingassing cycle to cease. This is owing to the fact that at $f \mathrm{O}_{2}$ $\sim$ QFM and at low pressures graphite becomes unstable and in equilibrium with pure $\mathrm{CO}_{2}$ vapor, $\mathrm{CO}_{2}$ solubility in mafic-ultramafic melts increases with pressure (Stolper and Holloway, 1988; Pan et al., 1991; Brooker et al., 2001). It is unclear at present whether towards the later part of the magma ocean crystallization $f \mathrm{O}_{2}$ became similar to that of QFM buffer. A recent study on Cerium oxidation state of zircon shows that at least in a continental crust-type environment, oxygen fugacity was similar to QFM buffer as early as $4.35 \mathrm{Ga}$ (Trail et al., 2011). If this oxygen fugacity condition is any reflection of $f \mathrm{O}_{2}$ towards the last phase of magma ocean crystallization, then the carbon pump has to cease. Therefore it is possible that magma ocean carbon ingassing may have operated within a window of time when coupled evolution of redox state and atmospheric chemistry was appropriate.

A recent paper by Hirschmann (2012) also speculated about the possibility of magma ocean carbon pump as a possible mechanism of enriching the bulk silicate Earth after core formation. However, the preferred model of Hirschmann (2012) called for the oxygen fugacity stratification of the magma ocean to be the primary driver of such a process. $\mathrm{Fe}^{3+}$ being more stable in higher pressure silicate melts of fixed and homogeneous $\mathrm{Fe}^{3+} / \mathrm{Fe}_{\text {total }}$ ratio, should facilitate carbon ingassing by having lower $\mathrm{CO}_{2}$ solubility of a graphite/diamond-saturated magma ocean at greater depths. However, the recent work of Righter and Ghiorso (2012a,b) showed that the trend of reduction with increasing depth for an isochemical magma ocean may not begin at pressures less than $20 \mathrm{GPa}$ and even at greater depths, the extent of reduction may be not so significant. Moreover, if the accreting material of the growing proto Earth became more and more FeO-rich (e.g., Wood et al., 2006), the magma ocean with depth up to $20 \mathrm{GPa}$ may even become slightly oxidized and remain essentially at a near-constant $\mathrm{fO}_{2}$ at greater depths (Righter and Ghiorso, 2012b); this is opposite to what is required for oxygen fugacity stratification-induced magma ocean carbon pump model of Hirschmann (2012). Therefore, if the absolute fugacity calculation of Righter and Ghiorso (2012a,b) truly captures the oxygen fugacity profiles of terrestrial magma oceans, then such gradients are not likely to be the primary driver of a carbon pump. Instead, pressure at a narrow range of or near-constant $f \mathrm{O}_{2}$, as proposed here, was likely a more important variable to facilitate ingassing of carbon through magma ocean-atmosphere interaction.

\subsubsection{Carbon outgassing from the core?}

All the possibilities of replenishing mantle carbon budget discussed thus far assume that there is no outgassing of core carbon since core formation. We speculate that this scenario may change after the initiation of plate tectonic cycles. If subduction of oceanic plates brings water to the core-mantle boundary regions, it may be possible that water, expelled from breakdown of hydrous phases, reacts with outer core metallic melt to form Fe-hydrides, $\mathrm{FeH}_{\mathrm{x}}$ (Okuchi, 1997, 1998) and oxygen released in the process reacts with carbon in the core to form $\mathrm{CO}_{2}, \mathrm{CO}$, or $\mathrm{FeCO}_{3}$, following a set of reactions such as:

$$
\begin{aligned}
& \mathrm{Fe}+\mathrm{H}_{2} \mathrm{O}=\mathrm{FeO}+2 \mathrm{H} \\
& \mathrm{Fe}+\mathrm{xH}=\mathrm{FeH}_{\mathrm{x}} \\
& \mathrm{FeO}+\mathrm{C}=\mathrm{Fe}+\mathrm{CO} \\
& 2 \mathrm{FeO}+\mathrm{C}=2 \mathrm{Fe}+\mathrm{CO}_{2} \\
& 2 \mathrm{FeO}+\mathrm{C}=2 \mathrm{Fe}+\mathrm{CO}_{2} \\
& \mathrm{FeO}+\mathrm{CO}_{2}=\mathrm{FeCO}_{3} \\
& \mathrm{C}+2 \mathrm{H}_{2}=\mathrm{CH}_{4}
\end{aligned}
$$

The outgassing of $\mathrm{CO}, \mathrm{CO}_{2}$, hydrocarbons, or carbonate, most likely as a solution component in a melt phase in D", from the core would follow, eventually causing the mantle carbon concentration to increase. Similar to the model of inefficient core formation, this possibility also can explain why the deeply sourced plume material may be more carbon-rich compared to shallower mantle.

\subsection{Carbon budget of the Earth's core through equilibrium core formation}

At our experimental conditions carbon behaves as a strongly siderophile element. But the equilibrium $\mathrm{C}$ content of the core depends not only on the $D_{\mathrm{C}}^{\text {metal/silicate }}$ and hence the conditions $\left(P-T-X-f \mathrm{O}_{2}\right)$ of metal-silicate equilibrium (e.g., Wood et al., 2006; Righter, 2011; Rubie et al., 2011) but also on the amount of $\mathrm{C}$ present at the time of equili- 
bration. If the dominant metal segregation occurred within a time frame when virtually all of the Earth's initial carbon budget was degassed to a thick proto atmosphere or had already been lost to space, the core could be essentially carbon-free. On the other hand, if substantial metal segregation preceded massive $\mathrm{C}$ outgassing, a C-bearing core may have formed. With our estimated $D_{\mathrm{C}}^{\mathrm{metal} / \text { silicate }}$, a bulk Earth carbon of 730 ppm generates a bulk core with $0.21-0.25$ wt.\% C (Fig. 9) and generating a core with $1 \mathrm{wt} . \%$ carbon requires the bulk Earth carbon of $\sim 3000$ ppm $\mathrm{C}$ participating in equilibrium core formation (Fig. 9).

\subsection{Core formation through incomplete equilibration of metal and silicate - Fate of carbon}

All the preceding discussions consider the magma ocean carbon cycle through a perfect equilibration of core and mantle forming liquids. However, some recent studies that considered Hf-W systematics (Halliday, 2004), geochemistry of Ni, Co, and W (Rubie et al., 2011), and the dynamics of accretion of differentiated large impactors on the proto Earth (Dahl and Stevenson, 2010), called for incomplete equilibration of segregating metallic liquid with the silicate magma ocean. Dahl and Stevenson (2010) argued that only $1-20 \%$ of the core mass might have equilibrated with the magma ocean. If this was the case, then one can envision the most extreme cases of core to mantle masses (1:210 $1: 10.5)$ relevant for equilibration. With such mass ratios, the magma ocean residual to core formation could retain $\sim 3(20 \%$ of core mass equilibrating with the magma ocean and with $D_{\mathrm{C}}^{\mathrm{metal} / \text { silicate }}$ of 2500$)$ to $216 \mathrm{ppm}$ ( $1 \%$ of cumulative core mass equilibrating with the magma ocean with $D_{\mathrm{C}}^{\text {metal/silicate }}$ of 500) carbon in dissolved form. Interestingly, in the latter case, i.e., if $1-2 \%$ of cumulative core mass equilibrated with the magma ocean, such a fraction of core liquid could reach carbon saturation value of $8 \mathrm{wt} . \%$ relevant for magma ocean temperatures ( $\geqslant 2273 \mathrm{~K}$; Fig. 3) and thus precipitate graphite or diamond in the process. Thus one might argue that this extremely imperfect coremantle equilibration scenario could eliminate the 'excess carbon' in the mantle problem by letting magma ocean hold onto a sizeable carbon budget in solution and by letting the segregating core liquid leave behind graphite or diamond (Hirschmann, 2012). However, we argue that this scenario is unlikely because if the core of a large differentiated impactor merges directly with the core of the proto Earth, the entire silicate mantle mass may not interact with the impactor. For example, the far side of the planet and/or the lower part of the mantle, which might have remained unmolten, would neither provide storage for carbon (Shcheka et al., 2006) nor allow an extreme mass ratio of metallic to silicate melt necessary to force metallic liquid into carbon saturation. Indeed, if a more modest extent of core-mantle equilibration $(36 \%$ of the core mass equilibrating with the mantle) as constrained recently by Rudge et al. (2010) is used, carbon saturation of segregating metallic melt would not occur. Lastly, the argument for core-mantle disequilibrium stems from the fact that the size of the metal blobs may exceed the length scale of diffusion of elements under consideration. However, the diffusion of carbon in magma ocean may be much faster than that of other elements and isotopes (e.g., Goldberg and Belton, 1974). Hence in a diffusion-based model, equilibration between metallic melt and silicate magma ocean with respect to carbon could have been more efficient. Therefore, even if emulsification of metallic melts to centimeter sized blobs did not occur, equilibrium carbon partitioning with metal meltsilicate melt mass ratio not too different from the relative masses of the mantle and core is likely expected.

\section{CONCLUDING REMARKS}

Our experiments suggest that carbon behaves as a siderophile element and $D_{\mathrm{C}}^{\text {metal/silicate }}$ increases with increasing pressure and decreases with increasing melt depolymerization. Thus if carbon content similar to that of the bulk Earth was available to participate in the early differentiation, most of it was partitioned to the core and $\leqslant 10-30 \%$ of the present-day mantle carbon budget could be derived from a magma ocean residual to core formation. Further studies exploring the effect of oxygen fugacity (even more reduced conditions of core-mantle differentiation), pressure (metal-silicate differentiation at greater depths), temperature, the presence and variable content of other light elements such as sulfur and silicon, in the metal, and known and variable content of other key volatile species such as hydrogen and sulfur, on $D_{\mathrm{C}}^{\mathrm{metal} / \text { silicate }}$ are needed to validate this conclusion. With equilibrium core formation removing most of the carbon initially retained in the terrestrial magma ocean, presumably at levels less than the carbonaceous chondrites, the modern Earth carbon inventory requires later replenishment mechanisms. Addition of volatile-rich, 'late veneer' and inefficient core formation both remain viable mechanisms. Alternatively, carbon ingassing by magma ocean-atmosphere interaction, after core formation, can also make the Earth's primordial carbon inventory similar to that of the present-day budget. We also speculate that reaction of subducted slab-bound water with outer core, in the core-mantle boundary regions, may cause formation of iron hydrides, thereby promoting outgassing oxidized and/or hydrocarbon forms of carbon. The hypotheses of inefficient core formation and core carbon leak through reaction with water can also explain the existence of carbon-rich deeper mantle domains, consistent with plumesource mantle coming from deeper depths being more carbon-rich.

\section{ACKNOWLEDGMENTS}

This work benefited from thoughtful reviews by Bruce Watson and an anonymous reviewer and comments from the journal Associate Editor Nicolas Dauphas. We thank Claude Herzberg, Liz Cottrell, and Kevin Righter for providing the peridotite xenolith, komatiite, and alkalic basalt powder, respectively. R.D. acknowledges support from a Lamont-Doherty Earth Observatory postdoctoral fellowship during early days of this study. A Packard fellowship for science and engineering to R.D. supported this work. D.W. and N.S. received support from NSF. 


\section{REFERENCES}

Abe Y. (1997) Thermal and chemical evolution of the terrestrial magma ocean. Phys. Earth Planet. Inter. 100, 27-39. http:// dx.doi.org/10.1016/s0031-9201(96)03229-3.

Anders E. and Grevesse N. (1989) Abundances of the elements: meteoritic and solar. Geochim. Cosmochim. Acta 53, 197-214.

Behrens H., Misiti V., Freda C., Vetere F., Botcharnikov R. E. and Scarlato P. (2009) Solubility of $\mathrm{H}_{2} \mathrm{O}$ and $\mathrm{CO}_{2}$ in ultrapotassic melts at 1200 and $1250^{\circ} \mathrm{C}$ and pressure from 50 to $500 \mathrm{MPa}$. Am. Mineral. 94, 105-120.

Blank J. G. and Brooker R. A. (1994) Experimental studies of carbon dioxide in silicate melts: solubility, speciation, and stable carbon isotope behavior. In Volatiles in Magmas (eds. M. R. Carroll and J. R. Holloway). Mineralogical Society of America, Washington, D.C.

Brooker R. A., Kohn S. C., Holloway J. R. and McMillan P. F. (2001) Structural controls on the solubility of $\mathrm{CO}_{2}$ in silicate melts Part I: bulk solubility data. Chem. Geol. 174, 225-239.

Chabot N. L., Draper D. S. and Agee C. B. (2005) Conditions of core formation in the Earth: constraints from nickel and cobalt partitioning. Geochim. Cosmochim. Acta 69, 2141-2151.

Chou C.-L. (1978) Fractionation of siderophile elements in the Earth's upper mantle. Proc. 9th Lunar Planet. Sci. Conf. 1, 219230.

Cottrell E. and Walker D. (2006) Constraints on core formation from Pt partitioning in mafic silicate liquids at high temperatures. Geochim. Cosmochim. Acta 70, 1565-1580.

Dahl T. W. and Stevenson D. J. (2010) Turbulent mixing of metal and silicate during planet accretion - and interpretation of the Hf-W chronometer. Earth Planet. Sci. Lett. 295, 177-186.

Dasgupta R., Buono A., Whelan G. and Walker D. (2009) Highpressure melting relations in $\mathrm{Fe}-\mathrm{C}-\mathrm{S}$ systems: implications for formation, evolution, and structure of metallic cores in planetary bodies. Geochim. Cosmochim. Acta 73, 6678-6691. http:// dx.doi.org/10.1016/j.gca.2009.08.001.

Dasgupta R. and Hirschmann M. M. (2010) The deep carbon cycle and melting in Earth's interior. Earth Planet. Sci. Lett. 298, 113. http://dx.doi.org/10.1016/j.epsl.2010.06.039.

Dasgupta R. and Walker D. (2008) Carbon solubility in core melts in a shallow magma ocean environment and distribution of carbon between the Earth's core and the mantle. Geochim. Cosmochim. Acta 72, 4627-4641. http://dx.doi.org/10.1016/ j.gca.2008.06.023.

Dauphas N. and Marty B. (2002) Inference on the nature and the mass of Earth's late veneer from noble metals and gases. $J$. Geophys. Res. 107, 5129. http://dx.doi.org/10.1029/ 2001JE001617.

Davis F. A., Tangeman J. A., Tenner T. J. and Hirschmann M. M. (2009) The composition of KLB-1 peridotite. Am. Mineral. 94, $176-180$.

Deines P. (2002) The carbon isotope geochemistry of mantle xenoliths. Earth Sci. Rev. 58, 247-278.

Dixon J. E., Stolper E. M. and Holloway J. R. (1995) An experimental study of water and carbon dioxide solubilities in mid-ocean ridge basaltic liquids. Part I: calibrations and solubility models. J. Petrol. 36, 1607-1631.

Eggler D. H. and Rosenhauer M. (1978) Carbon dioxide in silicate melts: II. Solubilities of $\mathrm{CO}_{2}$ and $\mathrm{H}_{2} \mathrm{O}$ in $\mathrm{CaMgSi}_{2} \mathrm{O}_{6}$ (diopside) liquids and vapors at pressures to $40 \mathrm{~kb}$. Am. J. Sci. 278, 64-94.

Elkins-Tanton L. T. (2008) Linked magma ocean solidification and atmospheric growth for Earth and Mars. Earth Planet. Sci. Lett. 271, 181-191.
Elkins-Tanton L. T. (2012) Magma Oceans in the Inner Solar System. Annu. Rev. Earth Planet. Sci. 40, 113-139. http:// dx.doi.org/10.1146/annurev-earth-042711-105503.

Filiberto J., Wood J., Dasgupta R., Shimizu N., Le L. and Treiman A. H. (2012) Effect of fluorine on near-liquidus phase equilibria of an Fe-Mg rich basalt. Chem. Geol. 312-313, 118-126. http:// dx.doi.org/10.1016/j.chemgeo.2012.04.015.

Genda H. and Abe Y. (2003) Survival of a proto-atmosphere through the stage of giant impacts: the mechanical aspects. Icarus 164, 149-162.

Goldberg D. and Belton G. (1974) The diffusion of carbon in ironcarbon alloys at $1560^{\circ} \mathrm{C}$. Metall. Mater. Trans. B 5, 1643-1648.

Halliday A. N. (2004) Mixing, volatile loss and compositional change during impact-driven accretion of the Earth. Nature 427, 505-509.

Helo C., Longpre M.-A., Shimizu N., Clague D. A. and Stix J. (2011) Explosive eruptions at mid-ocean ridges driven by $\mathrm{CO}_{2}-$ rich magmas. Nat. Geosci. 4, 260-263.

Hirschmann M. M. (2012) Magma ocean influence on early atmosphere mass and composition. Earth Planet. Sci. Lett. 341344, 48-57.

Hirschmann M. M. and Dasgupta R. (2009) The H/C ratios of Earth's near-surface and deep reservoirs, and consequences for deep Earth volatile cycles. Chem. Geol. 262, 4-16. http:// dx.doi.org/10.1016/j.chemgeo.2009.02.008.

Holloway J. R. and Jakobsson S. (1986) Volatile solubilities in magmas: transport of volatiles from mantles to planet surfaces. J. Geophys. Res. 91, D505-D508.

Holloway J. R., Pan V. and Gudmundsson G. (1992) Highpressure fluid-absent melting experiments in the presence of graphite; oxygen fugacity, ferric/ferrous ratio and dissolved $\mathrm{CO}_{2}$. Eur. J. Mineral. 4, 105-114.

Holzheid A., Palme H. and Chakraborty S. (1997) The activities of $\mathrm{NiO}, \mathrm{CoO}$ and $\mathrm{FeO}$ in silicate melts. Chem. Geol. 139, 21-38.

Jakobsson S. and Holloway J. R. (1986) Crystal-liquid experiments in the presence of a $\mathrm{C}-\mathrm{O}-\mathrm{H}$ fluid buffered by graphite + iron + wustite: experimental method and near-liquidus relations in basanite. J. Volcanol. Geotherm. Res. 29, 265-291.

Jana D. and Walker D. (1997) The impact of carbon in element distribution during core formation. Geochim. Cosmochim. Acta 61, 2759-2763.

Jones J. H. and Drake M. J. (1986) Geochemical constraints on core formation in the Earth. Nature 322, 221-228.

Kadik A., Pineau F., Litvin Y., Jendrzejewski N., Martinez I. and Javoy M. (2004) Formation of carbon and hydrogen species in magmas at low oxygen fugacity. J. Petrol. 45, 1297-1310.

Kadik A. A., Kurovskaya N. A., Ignat'ev Y. A., Kononkova N. N., Koltashev V. V. and Plotnichenko V. G. (2011) Influence of oxygen fugacity on the solubility of nitrogen, carbon, and hydrogen in $\mathrm{FeO}-\mathrm{Na}_{2} \mathrm{O}-\mathrm{SiO}_{2}-\mathrm{Al}_{2} \mathrm{O}_{3}$ melts in equilibrium with metallic iron at $1.5 \mathrm{GPa}$ and $1400^{\circ} \mathrm{C}$. Geochem. Int. 49, 429438.

Kasting J. (1993) Earth's early atmosphere. Science 259, 920-926.

Kegler P., Holzheid A., Frost D. J., Rubie D. C., Dohmen R. and Palme H. (2008) New Ni and Co metal-silicate partitioning data and their relevance for an early terrestrial magma ocean. Earth Planet. Sci. Lett. 268, 28-40.

Kerridge J. F. (1985) Carbon, hydrogen and nitrogen in carbonaceous chondrites: abundances and isotopic compositions in bulk samples. Geochim. Cosmochim. Acta 49, 1707-1714.

Kuramoto K. (1997) Accretion, core formation, H and C evolution of the Earth and Mars. Phys. Earth Planet. Inter. 100, 3-20.

Kuramoto K. and Matsui T. (1996) Partitioning of H and C between the mantle and core during the core formation in the Earth: its implications for the atmospheric evolution and redox state of early mantle. J. Geophys. Res. 101, 14909-14932. 
Lewis R. D., Lofgren G. E., Franzen H. F. and Windom K. E. (1993) The effect of $\mathrm{Na}$ vapor on the Na content of chondrules. Meteoritic 28, 622-628.

Li J. and Agee C. B. (1996) Geochemistry of mantle-core differentiation at high pressure. Nature 381, 686-689.

Lord O. T., Walter M. J., Dasgupta R., Walker D. and Clark S. M. (2009) Melting in the $\mathrm{Fe}-\mathrm{C}$ system to $70 \mathrm{GPa}$. Earth Planet. Sci. Lett. 284, 157-167.

Ma Z. (2001) Thermodynamic description for concentrated metallic solutions using interaction parameters. Metall. Mater. Trans. B 32, 87-103.

Mann U., Frost D. J. and Rubie D. C. (2008) The wetting ability of Si-bearing liquid Fe-alloys in a solid silicate matrix - percolation during core formation under reducing conditions? Phys. Earth Planet. Inter. 167, 1-7.

Marty B. (2012) The origins and concentrations of water, carbon, nitrogen and noble gases on Earth. Earth Planet. Sci. Lett. 313314, 56-66.

McDonough W. F. (2003) Compositional model for the Earth's core. In The Mantle and Core (ed. R. W. Carlson). ElsevierPergamon, Oxford.

Minarik W. G., Ryerson F. J. and Watson E. B. (1996) Textural Entrapment of core-forming melts. Science 272, 530-533.

Mysen B. O. (1988) Structure and Properties of Silicate Melts. Elsevier, Amsterdam.

Mysen B. O., Fogel M. L., Morrill P. L. and Cody G. D. (2009) Solution behavior of reduced $\mathrm{COH}$ volatiles in silicate melts at high pressure and temperature. Geochim. Cosmochim. Acta 73, 1696-1710.

Mysen B. O., Kumamoto K., Cody G. D. and Fogel M. L. (2011) Solubility and solution mechanisms of $\mathrm{C}-\mathrm{O}-\mathrm{H}$ volatiles in silicate melt with variable redox conditions and melt composition at upper mantle temperatures and pressures. Geochim. Cosmochim. Acta 75, 6183-6199.

Mysen B. O. and Yamashita S. (2010) Speciation of reduced C-O$\mathrm{H}$ volatiles in coexisting fluids and silicate melts determined insitu to $\sim 1.4 \mathrm{GPa}$ and $800{ }^{\circ} \mathrm{C}$. Geochim. Cosmochim. Acta 74, $4577-4588$.

Nakajima Y., Takahashi E., Toshihiro S. and Funakoshi K. (2009) "Carbon in the core" revisited. Phys. Earth Planet. Inter. 174, 202-211. http://dx.doi.org/10.1016/j.pepi.2008.05.014.

Newsom H. E. and Sims K. W. W. (1991) Core formation during early accretion of the Earth. Science 252, 926-933.

O'Neill H. S. C., Canil D. and Rubie D. C. (1998) Oxide-metal equilibria to $2500{ }^{\circ} \mathrm{C}$ and $25 \mathrm{GPa}$ : implications for core formation and the light component in the Earth's core. $J$. Geophys. Res. 103, 12239-12260.

O’Neill H. S. C. and Eggins S. M. (2002) The effect of melt composition on trace element partitioning: an experimental investigation of the activity coefficients of $\mathrm{FeO}, \mathrm{NiO}, \mathrm{CoO}$, $\mathrm{MoO}_{2}$ and $\mathrm{MoO}_{3}$ in silicate melts. Chem. Geol. 186, 151-181.

Okuchi T. (1997) Hydrogen partitioning into molten iron at high pressure: implications for Earth's core. Science 278, 1781-1784.

Okuchi T. (1998) The melting temperature of iron hydride at high pressures and its implications for the temperature of the Earth's core. J. Phys. Condens. Matter 10, 11595-11598.

Owen T. and Bar-Nun A. (1995) Comets, impacts, and atmospheres. Icarus 116, 215-226.

Palme H. and Jones A. (2003) Solar system abundances of the elements. In Meteorites, Comets, and Planets (ed. A. M. Davis). Elsevier Ltd., Amsterdam.

Pan V., Holloway J. R. and Hervig R. L. (1991) The pressure and temperature dependence of carbon dioxide solubility in tholeiitic basalt melts. Geochim. Cosmochim. Acta 55, 1587-1595.
Pawley A. R., Holloway J. R. and McMillan P. F. (1992) The effect of oxygen fugacity on the solubility of carbon-oxygen fluids in basaltic melt. Earth Planet. Sci. Lett. 110, 213-225.

Righter K. (2011) Prediction of metal-silicate partition coefficients for siderophile elements: an update and assessment of PT conditions for metal-silicate equilibrium during accretion of the Earth. Earth Planet. Sci. Lett. 304, 158-167.

Righter K. and Ghiorso M. S. (2012a) Redox systematics of a magma ocean with variable pressure-temperature gradients and composition. Proc. Nat. Acad. Sci. 109, 11955-11960.

Righter K. and Ghiorso M. S. (2012b) Correction for "Redox systematics of a magma ocean with variable pressure-temperature gradients and composition". Proc. Nat. Acad. Sci. 109, 16749-16750, http://dx.doi.org/10.1073/pnas.1214778109.

Rubie D. C., Frost D. J., Mann U., Asahara Y., Nimmo F., Tsuno K., Kegler P., Holzheid A. and Palme H. (2011) Heterogeneous accretion, composition and core-mantle differentiation of the Earth. Earth Planet. Sci. Lett. 301, 31-42.

Rudge J. F., Kleine T. and Bourdon B. (2010) Broad bounds on Earth's accretion and core formation constrained by geochemical models. Nat. Geosci. 3, 439-443.

Shannon M. C. and Agee C. B. (1996) High pressure constraints on percolative core formation. Geophys. Res. Lett. 23, 2717-2720.

Shcheka S. S., Wiedenbeck M., Frost D. J. and Keppler H. (2006) Carbon solubility in mantle minerals. Earth Planet. Sci. Lett. 245, 730-742.

Siebert J., Corgne A. and Ryerson F. J. (2011) Systematics of metal-silicate partitioning for many siderophile elements applied to Earth's core formation. Geochim. Cosmochim. Acta 75, 1451-1489.

Socrates G. (2004) Infrared and Raman Characteristic Group Frequencies: Tables and Charts. John Wiley and Sons, New York.

Stanley B. D., Hirschmann M. M. and Withers A. C. (2011) $\mathrm{CO}_{2}$ solubility in Martian basalts and Martian atmospheric evolution. Geochim. Cosmochim. Acta 75, 5987-6003.

Stevenson D. J. (1981) Models of the Earth's core. Science 214, 611-619.

Stolper E. and Holloway J. R. (1988) Experimental determination of the solubility of carbon dioxide in molten basalt at low pressure. Earth Planet. Sci. Lett. 87, 397-408.

Terasaki H., Frost D. J., Rubie D. C. and Langenhorst F. (2007) Interconnectivity of $\mathrm{Fe}-\mathrm{O}-\mathrm{S}$ liquid in polycrystalline silicate perovskite at lower mantle conditions. Phys. Earth Planet. Inter. 161, 170-176.

Thibault Y. and Holloway J. R. (1994) Solubility of $\mathrm{CO}_{2}$ in a Carich leucitite: effects of pressure, temperature, and oxygen fugacity. Contrib. Mineral. Petrol. 116, 216-224.

Thibault Y. and Walter M. J. (1995) The influence of pressure and temperature on the metal-silicate partition coefficients of nickel and cobalt in a model $\mathrm{Cl}$ chondrite and implications for metal segregation in a deep magma ocean. Geochim. Cosmochim. Acta 59, 991-1002.

Toplis M. J. (2005) The thermodynamics of iron and magnesium partitioning between olivine and liquid: criteria for assessing and predicting equilibrium in natural and experimental systems. Contrib. Mineral. Petrol. 149, 22-39. http://dx.doi.org/10.1007/ s00410-004-0629-4.

Trail D., Watson E. B. and Tailby N. D. (2011) The oxidation state of Hadean magmas and implications for early Earth's atmosphere. Nature 480, 79-82.

Tsuno K. and Dasgupta R. (2011) Melting phase relation of nominally anhydrous, carbonated pelitic-eclogite at 2.5 3.0 GPa and deep cycling of sedimentary carbon. Contrib. Mineral. Petrol. 161, 743-763. 
Wade J., Wood B. J. and Tuff J. (2012) Metal-silicate partitioning of Mo and $\mathrm{W}$ at high pressures and temperatures: evidence for late accretion of sulphur to the Earth. Geochim. Cosmochim. Acta 85, 58-74.

Wagner C. (1952) Thermodynamics of Alloys. Addison-Wesley Press.

Walter M. J. and Tronnes R. G. (2004) Early earth differentiation. Earth Planet. Sci. Lett. 225, 253-269.

Wänke H. (1981) Constitution of terrestrial planets. Philos. Trans. R. Soc. Lond. A 303, 287-302.

Watson H. C. and Roberts J. J. (2011) Connectivity of core forming melts: experimental constraints from electrical conductivity and X-ray tomography. Phys. Earth Planet. Inter. 186, 172-182.

Wetzel D. T., Jacobsen S. D., Rutherford M. J., Hauri E. H. and Saal A. E. (2012). The solubility and speciation of carbon in lunar picritic magmas. In 43rd Lunar Planet. Sci. Conf., Woodland, Texas.

Wood B. J. (1993) Carbon in the core. Earth Planet. Sci. Lett. 117, 593-607.

Wood B. J., Walter M. J. and Wade J. (2006) Accretion of the Earth and segregation of its core. Nature 441, 825-833.

Zahnle K., Arndt N., Cockell C., Halliday A., Nisbet E., Selsis F. and Sleep N. (2007) Emergence of a Habitable Planet. Space Sci. Rev. 129, 35-78.

Zahnle K., Schaefer L. and Fegley B. (2010) Earth's earliest atmospheres. Cold Spring Harbor Perspect. Biol. 2, a004895. http://dx.doi.org/10.1101/cshperspect.a004895.

Associate editor: Nicolas Dauphas 